\title{
Triply heavy tetraquark states
}

\author{
Xin-Zhen Weng $\oplus^{1,2, *}$ Wei-Zhen Deng, ${ }^{3, \dagger}$ and Shi-Lin Zhu $\oplus^{1, *}$ \\ ${ }^{1}$ Center of High Energy Physics, Peking University, Beijing 100871, China \\ ${ }^{2}$ School of Physics and Astronomy, Tel Aviv University, Tel Aviv 69978, Israel \\ ${ }^{3}$ School of Physics, Peking University, Beijing 100871, China
}

(Received 13 September 2021; accepted 9 February 2022; published 24 February 2022)

\begin{abstract}
In the framework of an extended chromomagnetic model, we systematically study the mass spectrum of the $S$-wave $q Q \bar{Q} \bar{Q}$ tetraquarks. Their mass spectra are mainly determined by the color interaction. For the $q c \bar{c} \bar{c}$, $q b \bar{c} \bar{c}$, and $q b \bar{b} \bar{b}$ tetraquarks, the color interaction favors the color-sextet $\left|(q Q)^{6_{c}}(\bar{Q} \bar{Q})^{\bar{\sigma}_{c}}\right\rangle$ configuration over the color-triplet $\left|(q Q)^{\overline{3}_{c}}(\bar{Q} \bar{Q})^{3_{c}}\right\rangle$ one. But for the $q c \bar{b} \bar{b}$ tetraquarks, the color-triplet configuration is favored. We find no stable states which lie below the thresholds of two pseudoscalar mesons. The lowest axial-vector states with the $q Q \bar{b} \bar{b}$ flavor configuration may be narrow. They lie just above the thresholds of two pseudoscalar mesons, but cannot decay into these channels because of the conservation of the angular momentum and parity.
\end{abstract}

DOI: 10.1103/PhysRevD.105.034026

\section{INTRODUCTION}

Searching for the exotic states is an important and challenging topic in hadron physics. In the past two decades, we have witnessed tremendous progresses in this area. In 2003, the first charmoniumlike state $X(3872)$ was observed by the Belle Collaboration [1], and later confirmed by the CDF [2], D0 [3], BABAR [4], LHCb [5], CMS [6], and BESIII [7] Collaborations. Its quantum number is $I^{G} J^{P C}=0^{+} 1^{++}$[8]. Since then, lots of charmoniumlike and bottomoniumlike states are found. They are called $X Y Z$ states. Many of them do not fit into the conventional charmonium or bottomonium spectrum in the quark model. In particular, the charged charmoniumlike and bottomoniumlike states like $Z_{c}(3900)$ [9,10], $Z_{c}(3885)$ [11,12], $Z_{c}(4020)$ [13], $Z_{c}(4025)$ [14], $Z_{c s}(3985)$ [15], $Z_{c s}(4000)$ and $Z_{c s}(4020)$ [16], $Z_{b}(10610)$ and $Z_{b}(10650)$ [17] contain at least four quarks. They are candidates of exotic structures like the compact tetraquark [18-22], the molecule [23-29], the hybrid meson [30,31], and so on. Interested readers may refer to Refs. [32-41] for more details.

Besides the charmoniumlike and bottomoniumlike states, various open heavy flavor exotic states have been

\footnotetext{
*xzhweng@pku.edu.cn

†dwz@pku.edu.cn

zhusl@pku.edu.cn
}

Published by the American Physical Society under the terms of the Creative Commons Attribution 4.0 International license. Further distribution of this work must maintain attribution to the author(s) and the published article's title, journal citation, and DOI. Funded by SCOAP. observed recently. In 2016, the D0 Collaboration reported the observation of the $X(5568)$ state in the $B_{s}^{0} \pi^{ \pm}$channel [42]. It was very likely a $u s \bar{d} \bar{b}$ or $d s \bar{u} \bar{b}$ tetraquarks since it lies about $200 \mathrm{MeV}$ below the $\bar{B} K$ threshold [43]. However, the subsequent search of the LHCb Collaboration did not confirm this state [44]. In 2020, the LHCb Collaboration observed two resonances in the $D^{-} K^{+}$channel with spin-0 and spin-1 $[45,46]$, whose minimal quark contents are $u d \bar{s} \bar{c}$. Recently, the LHCb Collaboration observed an narrow state in the $D^{0} D^{0} \pi^{+}$mass spectrum just below the $D^{*+} D^{0}$ threshold $[47,48]$. This is the first doubly charmed tetraquark $T_{c c}^{+}$observed in experiment. Moreover, in 2020, the LHCb collaboration observed a narrow structure and a wide structure in the $J / \psi$-pair invariant mass spectrum in the range of $6.2-7.2 \mathrm{GeV}$, which could be all-charm hadrons [49].

At this stage, it is natural to expect that the triply charmed tetraquark(s) may be observed in the very near future. For the triply charmed system, a molecule configuration requires the exchange of a $D$ meson. The corresponding interaction range is $\sim 0.1 \mathrm{fm}$, which is a typical scale of the compact tetraquark. From another point of view, the $D$-meson exchange interaction is highly suppressed in the typical scale of the molecule $(\sim \mathrm{fm})$. In other words, the molecule interpretation is not favored for the triply heavy system. If a $q c \bar{c} \bar{c}$ state is observed in experiment, it is very likely a compact tetraquark state.

However, the theoretical study of this system is relatively scarce [19,50-58]. In Ref. [52], Chen et al. found that some axial-vector $c c \bar{b} \bar{q}$ states lie below the $B_{c} \bar{D}_{(s)}$ thresholds, which are stable against the strong decay. A lattice study performed by Junnarkar et al. [54] gives similar results. They found that the axial-vector $u c \bar{b} \bar{b}$ and $s c \bar{b} \bar{b}$ lie below 
their thresholds by $6 \pm 11 \mathrm{MeV}$ and $8 \pm 3 \mathrm{MeV}$ respectively. However, a recent study by Lü et al. [58] predicted that the corresponding states are about several hundred $\mathrm{MeV}$ higher. More theoretical and experimental studies are needed to have a better understanding of these systems.

For the triply heavy tetraquarks, the interaction is provided by the gluon exchange and string confinement. The resulting interactions include the spin-independent Coulomb-type interaction, linear confinement and spindependent chromomagnetic interaction, tensor interaction, and spin-orbit interaction. When focusing on the $S$-wave states, the tensor and spin-orbit interactions can be neglected. Furthermore, we can simplify the model by integrating out spatial part of the interaction. Then the interaction becomes

$$
-\sum_{i<j} a_{i j} \boldsymbol{F}_{i} \cdot \boldsymbol{F}_{j}-\sum_{i<j} v_{i j} \boldsymbol{S}_{i} \cdot \boldsymbol{S}_{j} \boldsymbol{F}_{i} \cdot \boldsymbol{F}_{j}
$$

This interaction gives good account of the $S$-wave mesons and baryons [59]. In this work, we use this interaction to study the triply heavy tetraquarks. The paper is organized as follows. In Sec. II, we introduce the extended chromomagnetic model and construct the wave function bases for the triply heavy tetraquarks. Then we discuss the numerical results in Sec. III. We conclude in Sec. IV.

\section{THE EXTENDED CHROMOMAGNETIC MODEL}

For the $S$-wave tetraquark system, we consider the chromomagnetic model. The Hamiltonian reads [59-67]

$$
H=\sum_{i} m_{i}+H_{\mathrm{CE}}+H_{\mathrm{CM}}
$$

where $m_{i}$ is the effective mass of $i$ th constituent quark which consists of the constituent quark mass, the kinetic energy, and so on, $H_{\mathrm{CE}}$ is the colorelectric (CE) interaction which includes the color linear confinement and Coulombtype interaction [59,64-67]

$$
H_{\mathrm{CE}}=-\sum_{i<j} a_{i j} \boldsymbol{F}_{i} \cdot \boldsymbol{F}_{j},
$$

and $H_{\mathrm{CM}}$ is the chromomagnetic $(\mathrm{CM})$ interaction from the one-gluon-exchange (OGE) [19,40,52,68-71]

$$
H_{\mathrm{CM}}=-\sum_{i<j} v_{i j} \boldsymbol{S}_{i} \cdot \boldsymbol{S}_{j} \boldsymbol{F}_{i} \cdot \boldsymbol{F}_{j} .
$$

The coupling constants $a_{i j}$ and $v_{i j} \propto\left\langle\alpha_{s}(r) \delta^{3}(\boldsymbol{r})\right\rangle / m_{i} m_{j}$ depend on the spatial wave function and the constituent quark masses. $\boldsymbol{S}_{i}=\boldsymbol{\sigma}_{i} / 2$ and $\boldsymbol{F}_{i}=\boldsymbol{\lambda}_{i} / 2$ are the quark spin and color operators. For the antiquark,

$$
\boldsymbol{S}_{\bar{q}}=-\boldsymbol{S}_{q}^{*}, \quad \boldsymbol{F}_{\bar{q}}=-\boldsymbol{F}_{q}^{*} .
$$

Since

$$
\begin{aligned}
& \sum_{i<j}\left(m_{i}+m_{j}\right) \boldsymbol{F}_{i} \cdot \boldsymbol{F}_{j} \\
& =\left(\sum_{i} m_{i} \boldsymbol{F}_{i}\right) \cdot\left(\sum_{i} \boldsymbol{F}_{i}\right)-\frac{4}{3} \sum_{i} m_{i},
\end{aligned}
$$

and the total color operator $\sum_{i} \boldsymbol{F}_{i}$ nullifies any color-singlet physical state, we introduce the quark pair mass parameter

$$
m_{i j}=\left(m_{i}+m_{j}\right)+\frac{4}{3} a_{i j},
$$

to combine the effective quark mass $m_{i}$ and the color interaction strength $a_{i j}$. Then we can rewrite the model Hamiltonian as [59,65-67]

$$
H=-\frac{3}{4} \sum_{i<j} m_{i j} V_{i j}^{\mathrm{C}}-\sum_{i<j} v_{i j} V_{i j}^{\mathrm{CM}},
$$

where

$$
V_{i j}^{\mathrm{C}}=\boldsymbol{F}_{i} \cdot \boldsymbol{F}_{j},
$$

and

$$
V_{i j}^{\mathrm{CM}}=\boldsymbol{S}_{i} \cdot \boldsymbol{S}_{j} \boldsymbol{F}_{i} \cdot \boldsymbol{F}_{j}
$$

are the color and CM interactions between quarks, respectively. Here, $m_{i j}$ and $v_{i j}$ are unknown parameters. In Ref. [59], we have used the conventional mesons and baryons to fit them. More precisely, we used the pseudoscalar and vector mesons to extract the parameters $m_{q \bar{q}}$ and $v_{q \bar{q}}$, and used the light and singly heavy baryons to fit the $\left\{m_{q q}, v_{q q}\right\}$ with at most one heavy quark, estimated the $m_{Q Q}$ and $v_{Q Q}$ through a quark model consideration. Their values are listed in Table I. With these parameters, we reproduce the meson and baryon masses with errors mostly within $10 \mathrm{MeV}$ (The only exception is the $\Sigma$ baryon, whose deviation is $15 \mathrm{MeV}$.). We also obtained the $\Xi_{c c}$ baryon mass very close to the LHCb Collaboration's measurement $\left(M_{\text {th. }}=3633.3 \pm 9.3 \mathrm{MeV}\right.$ versus $\left.M_{\text {exp. }}=3621.55 \pm 0.23 \pm 0.30 \mathrm{MeV}\right)[72,73]$. In Ref. [66], we used these parameters to study the fully heavy tetraquark systems. We found that for the ground states, the color sextet component is more important than the color triplet one, which is consistent with the dynamical calculations $[74,75]$. We also studied the hidden-charm pentaquark states, and successfully reproduced the four $P_{c}$ states, $P_{c}$ (4312), $P_{c}$ (4380), $P_{c}(4440)$, and $P_{c}(4450)[76,77]$ with these parameters [65]. In this work, we use the same set of parameters to estimate the masses of the $S$-wave $q Q \bar{Q} \bar{Q}$ tetraquarks. 
TABLE I. Parameters of the $q \bar{q}$ and $q q$ pairs [59] (in units of $\mathrm{MeV}$ ).

\begin{tabular}{lcccccccccc}
\hline \hline Parameter & $m_{n \bar{n}}$ & $m_{n \bar{s}}$ & $m_{s \bar{s}}$ & $m_{n \bar{c}}$ & $m_{s \bar{c}}$ & $m_{c \bar{c}}$ & $m_{n \bar{b}}$ & $m_{s \bar{b}}$ & $m_{c \bar{b}}$ & $m_{b \bar{b}}$ \\
\hline Value & 615.95 & 794.22 & 936.40 & 1973.22 & 2076.14 & 3068.53 & 5313.35 & 5403.25 & 6322.27 & 9444.97 \\
Parameter & $v_{n \bar{n}}$ & $v_{n \bar{s}}$ & $v_{s \bar{s}}$ & $v_{n \bar{c}}$ & $v_{s \bar{c}}$ & $v_{c \bar{c}}$ & $v_{n \bar{b}}$ & $v_{s \bar{b}}$ & $v_{c \bar{b}}$ & $v_{b \bar{b}}$ \\
Value & 477.92 & 298.57 & 249.18 & 106.01 & 107.87 & 85.12 & 33.89 & 36.43 & 47.18 & 45.98 \\
\hline \hline Parameter & $m_{n n}$ & $m_{n s}$ & $m_{s s}$ & $m_{n c}$ & $m_{s c}$ & $m_{c c}$ & $m_{n b}$ & $m_{s b}$ & $m_{c b}$ & $m_{b b}$ \\
\hline Value & 724.85 & 906.65 & 1049.36 & 2079.96 & 2183.68 & 3171.51 & 5412.25 & 5494.80 & 6416.07 & 9529.57 \\
Parameter & $v_{n n}$ & $v_{n s}$ & $v_{s s}$ & $v_{n c}$ & $v_{s c}$ & $v_{c c}$ & $v_{n b}$ & $v_{s b}$ & $v_{c b}$ & $v_{b b}$ \\
Value & 305.34 & 212.75 & 195.30 & 62.81 & 70.63 & 56.75 & 19.92 & 8.47 & 31.45 & 30.65 \\
\hline \hline
\end{tabular}

\section{A. Wave function}

Before calculating the tetraquark masses, we need to construct their wave functions. In principle, the total wave function is a direct product of the orbital, color, spin, and flavor wave functions. In this work, we only consider the $S$-wave states, the orbital wave function is always symmetric. In the $q q \otimes \bar{q} \bar{q}$ configuration, we can construct the following color-spin wave functions $\left\{\alpha_{i}^{J}\right\}$

(1) $J^{P}=0^{+}$:

$$
\begin{aligned}
& \alpha_{1}^{0}=\left|\left(q_{1} q_{2}\right)_{1}^{6}\left(\bar{q}_{3} \bar{q}_{4}\right)_{1}^{\overline{6}}\right\rangle_{0}, \\
& \alpha_{2}^{0}=\left|\left(q_{1} q_{2}\right)_{0}^{6}\left(\bar{q}_{3} \bar{q}_{4}\right)_{0}^{\overline{6}}\right\rangle_{0}, \\
& \alpha_{3}^{0}=\left|\left(q_{1} q_{2}\right)_{1}^{\overline{3}}\left(\bar{q}_{3} \bar{q}_{4}\right)_{1}^{3}\right\rangle_{0}, \\
& \alpha_{4}^{0}=\left|\left(q_{1} q_{2}\right)_{0}^{3}\left(\bar{q}_{3} \bar{q}_{4}\right)_{0}^{3}\right\rangle_{0},
\end{aligned}
$$

(2) $J^{P}=1^{+}$:

$$
\begin{aligned}
\alpha_{1}^{1} & =\left|\left(q_{1} q_{2}\right)_{1}^{6}\left(\bar{q}_{3} \bar{q}_{4}\right)_{1}^{\overline{6}}\right\rangle_{1}, \\
\alpha_{2}^{1} & =\left|\left(q_{1} q_{2}\right)_{1}^{6}\left(\bar{q}_{3} \bar{q}_{4}\right)_{0}^{6}\right\rangle_{1}, \\
\alpha_{3}^{1} & =\left|\left(q_{1} q_{2}\right)_{0}^{6}\left(\bar{q}_{3} \bar{q}_{4}\right)_{1}^{\overline{6}}\right\rangle_{1}, \\
\alpha_{4}^{1} & =\left|\left(q_{1} q_{2}\right)_{1}^{\overline{3}}\left(\bar{q}_{3} \bar{q}_{4}\right)_{1}^{3}\right\rangle_{1}, \\
\alpha_{5}^{1} & =\left|\left(q_{1} q_{2}\right)_{1}^{3}\left(\bar{q}_{3} \bar{q}_{4}\right)_{0}^{3}\right\rangle_{1}, \\
\alpha_{6}^{1} & =\left|\left(q_{1} q_{2}\right)_{0}^{\overline{3}}\left(\bar{q}_{3} \bar{q}_{4}\right)_{1}^{3}\right\rangle_{1},
\end{aligned}
$$

(3) $J^{P}=2^{+}$:

$$
\begin{aligned}
& \alpha_{1}^{2}=\left|\left(q_{1} q_{2}\right)_{1}^{6}\left(\bar{q}_{3} \bar{q}_{4}\right)_{1}^{\overline{6}}\right\rangle_{2}, \\
& \alpha_{2}^{2}=\left|\left(q_{1} q_{2}\right)_{1}^{\overline{3}}\left(\bar{q}_{3} \bar{q}_{4}\right)_{1}^{3}\right\rangle_{2},
\end{aligned}
$$

where the superscript $3, \overline{3}, 6$ or $\overline{6}$ denotes the color, and the subscript 0,1 or 2 denotes the spin.

Next we consider the flavor wave functions. According to symmetric properties of the two antiquarks, the triply heavy tetraquarks can be divided into two categories. The $q Q \bar{c} \bar{b}$ tetraquarks are not constrained by the Pauli principle, thus all the preceding color-spin wave functions are allowed. On the other hand, the $q Q \bar{c} \bar{c}$ and $q Q \bar{b} \bar{b}$ tetraquarks have symmetric flavor wave functions over the two antiquarks, thus the color-spin wave functions must be asymmetric over the two antiquarks. More precisely, the bases for the $q Q \bar{c} \bar{c}$ and $q Q \bar{b} \bar{b}$ tetraquarks are $\left\{\alpha_{2}^{0}, \alpha_{3}^{0}\right\}$, $\left\{\alpha_{2}^{1}, \alpha_{4}^{1}, \alpha_{4}^{1}\right\}$ and $\left\{\alpha_{2}^{2}\right\}$.

Diagonalizing the Hamiltonian [Eq. (8)] in the corresponding bases, we can obtain the masses and eigenvectors of the triply heavy tetraquarks.

\section{NUMERICAL RESULTS}

\section{A. The $q c \bar{c} \bar{c}$ and $q b \bar{b} \bar{c}$ systems}

First we consider the $n c \bar{c} \bar{c}$ and $s c \bar{c} \bar{c}$ tetraquarks. Their masses and eigenvectors are listed in Tables II. For simplicity, we will use $T\left(q Q \bar{Q} \bar{Q}, m, J^{P}\right)$ to denote the $q Q \bar{Q} \bar{Q}$ tetraquarks in the following. The isospin of the tetraquarks can be easily identified with either $q=n$ or $q=s$. Here we assume that the $\mathrm{SU}(2)$ flavor symmetry is exact and $n=\{u, d\}$. We plot their relative position in Fig 1, along with the corresponding meson-meson thresholds. From the figure, we can easily see that the quantum number of the ground states are $J^{P}=0^{+}$in both cases.

TABLE II. Masses and eigenvectors of the $n c \bar{c} \bar{c}$ and $s c \bar{c} \bar{c}$ tetraquarks. The masses are all in units of $\mathrm{MeV}$.

\begin{tabular}{llcc}
\hline \hline System & $J^{P}$ & Mass & Eigenvector \\
\hline$n c \bar{c} \bar{c}$ & $0^{+}$ & 4936.7 & $\{0.817,0.576\}$ \\
& & 5185.3 & $\{-0.576,0.817\}$ \\
& $1^{+}$ & 4968.1 & $\{0.915,-0.069,-0.397\}$ \\
& & 5135.5 & $\{-0.071,-0.997,0.010\}$ \\
& & 5154.0 & $\{-0.397,0.019,-0.918\}$ \\
& $2^{+}$ & 5198.4 & $\{1\}$ \\
$s c \bar{c} \bar{c}$ & $0^{+}$ & 5040.1 & $\{0.816,0.578\}$ \\
& & 5290.6 & $\{-0.578,0.816\}$ \\
& $1^{+}$ & 5069.2 & $\{0.912,-0.074,-0.404\}$ \\
& & 5239.9 & $\{-0.076,-0.997,0.011\}$ \\
& & 5254.3 & $\{-0.404,0.020,-0.915\}$ \\
& $2^{+}$ & 5303.3 & $\{1\}$ \\
\hline \hline
\end{tabular}



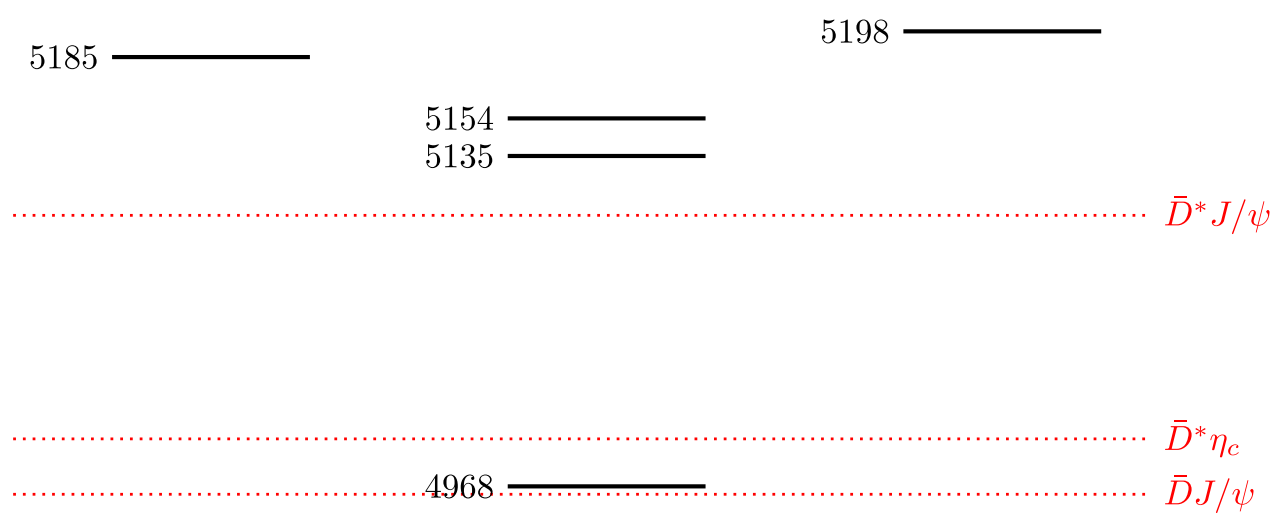

4937

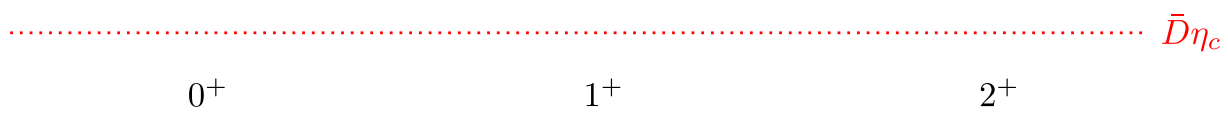

(a) $n c \bar{c} \bar{c}$ states

5291

5303

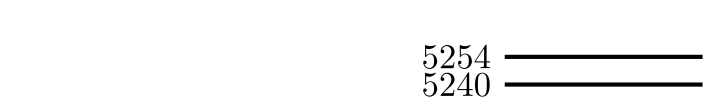

$\bar{D}_{s}^{*} J / \psi$

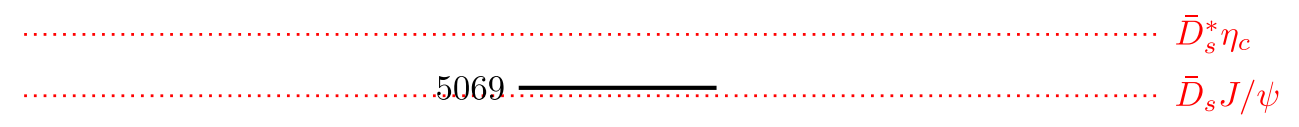

5040

$\bar{D}_{s} \eta_{c}$

$0^{+}$

$1^{+}$

$2^{+}$

(b) $s c \bar{c} \bar{c}$ states

FIG. 1. Mass spectra of the $n c \bar{c} \bar{c}$ and $s c \bar{c} \bar{c}$ tetraquark states. The dotted lines indicate various meson-meson thresholds. The masses are all in units of $\mathrm{MeV}$.

They are $T\left(n c \bar{c} \bar{c}, 4936.7,0^{+}\right)$and $T\left(s c \bar{c} \bar{c}, 5040.1,0^{+}\right)$, respectively. On the other hand, the highest mass states have quantum number $J^{P}=2^{+}$. Comparing to the thresholds, we find that all states are above the meson-meson thresholds and can decay through $S$-wave. They may all be broad states [68]. The lowest axial-vector states $T\left(n c \bar{c} \bar{c}, 4968.1,1^{+}\right) / T\left(s c \bar{c} \bar{c}, 5069.2,1^{+}\right)$lie just above the $D_{(s)} J / \psi$ threshold. Thus they might be relatively narrow compared to other states.

Besides the masses, the eigenvectors also provide important information of the tetraquarks. The color configurations of the tetraquarks can be of $\left|\left(q_{1} q_{2}\right)^{6_{c}}\left(\bar{q}_{3} \bar{q}_{4}\right)^{\overline{6}_{c}}\right\rangle$ or $\left|\left(q_{1} q_{2}\right)^{\overline{3}_{c}}\left(\bar{q}_{3} \bar{q}_{4}\right)^{3_{c}}\right\rangle$. For simplicity, we denote them as $6_{c} \otimes \overline{6}_{c}$ and $\overline{3}_{c} \otimes 3_{c}$. From Table II, we find that the 
ground states of the $n c \bar{c} \bar{c}$ and $s c \bar{c} \bar{c}$ tetraquarks are both dominated by the color-sextet components. The $T\left(n c \bar{c} \bar{c}, 4936.7,0^{+}\right)$has $66.7 \%$ of the $6_{c} \otimes \overline{6}_{c}$ component and the $T\left(\operatorname{sc} \bar{c} \bar{c}, 5040.1,0^{+}\right)$has $66.6 \%$. This is very similar to the fully charm tetraquarks $[66,74,75,78]$. In the onegluon-exchange (OGE) model, the color interactions are attractive inside $\left(q_{1} q_{2}\right)^{\overline{3}_{c}} /\left(\bar{q}_{3} \bar{q}_{4}\right)^{3_{c}}$ and repulsive inside $\left(q_{1} q_{2}\right)^{6_{c}} /\left(\bar{q}_{3} \bar{q}_{4}\right)^{\overline{6}_{c}}$. However, the attractions between $\left(q_{1} q_{2}\right)^{6_{c}}$ and $\left(\bar{q}_{3} \bar{q}_{4}\right)^{\overline{6}_{c}}$ is much stronger than that between $\left(q_{1} q_{2}\right)^{\overline{3}_{c}}$ and $\left(\bar{q}_{3} \bar{q}_{4}\right)^{3_{c}}$. In our cases, the two competing effects result in the lower mass of the color-sextet configurations. More precisely,

$$
\begin{aligned}
\left\langle H_{\mathrm{C}}(q c \bar{c} \bar{c})\right\rangle= & -\frac{3}{4}\left\langle m_{q c} V_{12}^{\mathrm{C}}+m_{c c} V_{34}^{\mathrm{C}}+m_{q \bar{c}}\left(V_{13}^{\mathrm{C}}+V_{14}^{\mathrm{C}}\right)\right. \\
& \left.+m_{c \bar{c}}\left(V_{23}^{\mathrm{C}}+V_{24}^{\mathrm{C}}\right)\right\rangle \\
= & m_{q \bar{c}}+m_{c \bar{c}}-\frac{3}{2} \delta m\left\langle V_{12}^{\mathrm{C}}+V_{34}^{\mathrm{C}}\right\rangle \\
= & m_{q \bar{c}}+m_{c \bar{c}}+\delta m\left(\begin{array}{cc}
-1 & 0 \\
0 & +2
\end{array}\right)
\end{aligned}
$$

where $\delta m=\left(m_{q c}+m_{c c}-m_{q \bar{c}}-m_{c \bar{c}}\right) / 4$. Inserting the parameters in Table I, we have

$$
\begin{aligned}
& \delta m(n c \bar{c} \bar{c})=52.43 \mathrm{MeV}, \\
& \delta m(s c \bar{c} \bar{c})=52.63 \mathrm{MeV} .
\end{aligned}
$$

Similar to the fully charm cases, the color interaction renders the $\sigma_{c} \otimes \bar{\sigma}_{c}$ configuration more stable than the $\overline{3}_{c} \otimes 3_{c}$ one. We also find that the color interaction does not mix the two color configurations. This is because the two antiquarks are identical $[67,74]$. However, the two color configuration will mix due to the $\mathrm{CM}$ interaction

$$
\begin{aligned}
& \left\langle H_{\mathrm{CM}}(q c \bar{c} \bar{c})\right\rangle \\
& =\left(\begin{array}{cc}
\frac{1}{4}\left(v_{q c}+v_{c c}\right) & -\frac{\sqrt{6}}{4}\left(v_{q \bar{c}}+v_{c \bar{c}}\right) \\
-\frac{\sqrt{6}}{4}\left(v_{q \bar{c}}+v_{c \bar{c}}\right) & \frac{1}{6}\left(v_{q c}+v_{c c}\right)-\frac{1}{3}\left(v_{q \bar{c}}+v_{c \bar{c}}\right)
\end{array}\right) \\
& \approx \frac{v_{q \bar{c}}+v_{c \bar{c}}}{2}\left(\begin{array}{cc}
\frac{1}{3} & -\sqrt{\frac{3}{2}} \\
-\sqrt{\frac{3}{2}} & -\frac{4}{9}
\end{array}\right)
\end{aligned}
$$

where we have used $v_{q_{1} q_{2}} / v_{q_{1} \bar{q}_{2}} \approx 2 / 3$ in the last line [59]. Here, $\left(v_{n \bar{c}}+v_{c \bar{c}}\right) / 2=95.57 \mathrm{MeV}$ and $\left(v_{s \bar{c}}+v_{c \bar{c}}\right) / 2=$ $96.50 \mathrm{MeV}$. The diagonal part of the CM interaction favors the $\overline{3}_{c} \otimes 3_{c}$ configuration by approximately $75 \mathrm{MeV}$. However, Eq. (14) indicates that the color interaction disfavor the $\overline{3}_{c} \otimes 3_{c}$ configuration by approximately $150 \mathrm{MeV}$. Thus the ground states of the scalar $q c \bar{c} \bar{c}$ tetraquarks are dominated by the $\sigma_{c} \otimes \bar{\sigma}_{c}$ component, while the higher scalar states mostly consist of $\overline{3}_{c} \otimes 3_{c}$ component.

There are three bases for the axial-vector states, namely $\left|(q c)_{1}^{6}(\bar{c} \bar{c})_{0}^{\overline{6}}\right\rangle, \quad\left|(q c)_{1}^{\overline{3}}(\bar{c} \bar{c})_{1}^{3}\right\rangle$ and $\left|(q c)_{0}^{\overline{3}}(\bar{c} \bar{c})_{1}^{3}\right\rangle$. In these bases, we have

$$
\left\langle H_{\mathrm{C}}(q c \bar{c} \bar{c})\right\rangle=m_{q \bar{c}}+m_{c \bar{c}}+\delta m\left(\begin{array}{ccc}
-1 & 0 & 0 \\
0 & +2 & 0 \\
0 & 0 & +2
\end{array}\right)
$$

The color interaction splits the three bases into two energy bands. The $6_{c} \otimes \overline{6}_{c}$ configuration is more stable than the $\overline{3}_{c} \otimes 3_{c}$. The CM interaction further splits the two bands into the three bands structure in Fig. 1. As a consequence, the lightest axial-vector states of both $n c \bar{c} \bar{c}$ and $s c \bar{c} \bar{c}$ tetraquarks have more than $80 \%$ of the $\sigma_{c} \otimes \bar{\sigma}_{c}$ component. Combining the scalar and axial-vector cases, we can conclude that the tetraquark spectrum is dominantly determined by the color interaction, and the $\mathrm{CM}$ interaction contributes to the finer structures.

The eigenvectors can also be used to study the decay properties of the tetraquarks. We can transform the wave functions into the $q \bar{q} \otimes q \bar{q}$ configuration. The corresponding color configuration can be either of $\left|(q \bar{q})^{1_{c}}(q \bar{q})^{1_{c}}\right\rangle$ or $\left|(q \bar{q})^{8_{c}}(q \bar{q})^{8_{c}}\right\rangle$. The former one can easily decay into two $S$-wave mesons in $S$ wave (the so-called "Okubo-ZweigIizuka- (OZI-)superallowed" decays), while the latter one can fall apart only through gluon exchange. Following Refs. $[68,69,79]$, we only consider the "OZI-superallowed" decays. In Tables III-IV, we transform the eigenvectors of the $q c \bar{c} \bar{c}$ tetraquarks into the $q \bar{c} \otimes c \bar{c}$ configuration. For simplicity, we only present the color-singlet components,

TABLE III. The eigenvectors of the $n c \bar{c} \bar{c}$ tetraquarks in the $n \bar{c} \otimes c \bar{c}$ configuration. The masses are all in units of $\mathrm{MeV}$.

\begin{tabular}{lccrrrr}
\hline \hline System & $J^{P}$ & Mass & $\bar{D}^{*} J / \psi$ & $\bar{D}^{*} \eta_{c}$ & $\bar{D} J / \psi$ & $\bar{D} \eta_{c}$ \\
\hline$n c \bar{c} \bar{c}$ & $0^{+}$ & 4936.7 & 0.412 & & & 0.622 \\
& & 5185.3 & -0.643 & & & 0.174 \\
& $1^{+}$ & 4968.1 & 0.366 & 0.460 & -0.516 & \\
& & 5135.5 & -0.037 & -0.439 & -0.375 & \\
& & 5154.0 & -0.604 & 0.111 & -0.095 & \\
& $2^{+}$ & 5198.4 & 0.577 & & & \\
\hline \hline
\end{tabular}

TABLE IV. The eigenvectors of the $s c \bar{c} \bar{c}$ tetraquarks in the $s \bar{c} \otimes c \bar{c}$ configuration. The masses are all in units of $\mathrm{MeV}$.

\begin{tabular}{lccrrrr}
\hline \hline System & $J^{P}$ & Mass & $\bar{D}_{s}^{*} J / \psi$ & $\bar{D}_{s}^{*} \eta_{c}$ & $\bar{D}_{s} J / \psi$ & $\bar{D}_{s} \eta_{c}$ \\
\hline$s c \bar{c} \bar{c}$ & $0^{+}$ & 5040.1 & 0.410 & & & 0.622 \\
& & 5290.6 & -0.644 & & & 0.172 \\
& $1^{+}$ & 5069.2 & 0.361 & 0.459 & -0.519 & \\
& & 5239.9 & -0.039 & -0.441 & -0.373 & \\
& & 5254.3 & -0.607 & 0.107 & -0.091 & \\
& $2^{+}$ & 5303.3 & 0.577 & & & \\
\hline \hline
\end{tabular}


and we rewrite the bases as a direct product of two mesons. For each decay channel, the decay width is proportional to the square of the coefficient $c_{i}$ of the corresponding component in the eigenvectors, and also depends on the phase space. For two body decay through $L$-wave, the partial decay width reads $[65,80]$

$$
\Gamma_{i}=\gamma_{i} \alpha \frac{k^{2 L+1}}{m^{2 L}} \cdot\left|c_{i}\right|^{2},
$$

where $\gamma_{i}$ is a quantity determined by the decay dynamics, $\alpha$ is an effective coupling constant, $k$ is the momentum of the final states in the rest frame of the initial state, and $m$ is the mass of the initial state. In this work, the $(\mathrm{k} / \mathrm{m})^{2}$ is always of $\mathcal{O}\left(10^{-2}\right)$ or even smaller. Thus we will only consider the $S$-wave decays. Next we consider the $\gamma_{i}$. Generally, $\gamma_{i}$ depends on the spatial wave functions of the initial tetraquark and final mesons, which are different for each decay process. In the quark model, the spatial wave functions of the ground state scalar and axial-vector meson are the same $[81,82]$. Thus for each tetraquark, we have

$$
\gamma_{M_{1} M_{2}}=\gamma_{M_{1} M_{2}^{*}}=\gamma_{M_{1}^{*} M_{2}}=\gamma_{M_{1}^{*} M_{2}^{*}}
$$

where $M_{i}$ and $M_{i}^{*}$ denote the pseudoscalar and axial-vector mesons. With the eigenvectors obtained, we calculate the value of $k \cdot\left|c_{i}\right|^{2}$ and the relative widths for the $q c \bar{c} \bar{c}$ decays, as shown in Tables V-VIII. Their ground states can easily decay into $\bar{D}_{(s)} J / \psi$, thus may be broad states [68]. The higher scalar states, $T\left(n c \bar{c} \bar{c}, 5185.3,0^{+}\right) /$ $T\left(s c \bar{c} \bar{c}, 5290.6,0^{+}\right)$, can decay to $\bar{D}_{(s)}^{*} J / \psi$ and $\bar{D}_{(s)} J / \psi$ modes through $S$-wave, with relative decay width ratios

TABLE V. The values of $k \cdot\left|c_{i}\right|^{2}$ for the $n c \bar{c} \bar{c}$ tetraquarks (in units of $\mathrm{MeV}$ ).

\begin{tabular}{lcccccc}
\hline \hline System & $J^{P}$ & Mass & $\bar{D}^{*} J / \psi$ & $\bar{D}^{*} \eta_{c}$ & $\bar{D} J / \psi$ & $\bar{D} \eta_{c}$ \\
\hline$n c \bar{c} \bar{c}$ & $0^{+}$ & 4936.7 & $\times$ & & & 172.8 \\
& & 5185.3 & 183.4 & & & 27.0 \\
& $1^{+}$ & 4968.1 & $\times$ & $\times$ & 25.9 & \\
& & 5135.5 & 0.4 & 114.1 & 90.0 & \\
& & 5154.0 & 125.7 & 7.7 & 6.1 & \\
& $2^{+}$ & 5198.4 & 159.5 & & & \\
\hline \hline
\end{tabular}

TABLE VI. The values of $k \cdot\left|c_{i}\right|^{2}$ for the $s c \bar{c} \bar{c}$ tetraquarks (in units of $\mathrm{MeV}$ ).

\begin{tabular}{lcccccr}
\hline \hline System & $J^{P}$ & Mass & $\bar{D}_{s}^{*} J / \psi$ & $\bar{D}_{s}^{*} \eta_{c}$ & $\bar{D}_{s} J / \psi$ & $\bar{D}_{s} \eta_{c}$ \\
\hline$s c \bar{c} \bar{c}$ & $0^{+}$ & 5040.1 & $\times$ & & & 178.2 \\
& & 5290.6 & 188.8 & & & 27.0 \\
& $1^{+}$ & 5069.2 & $\times$ & $\times$ & 26.4 & \\
& & 5239.9 & 0.4 & 117.3 & 91.1 & \\
& & 5254.3 & 124.4 & 7.3 & 5.6 & \\
& $2^{+}$ & 5303.3 & 163.1 & & & \\
\hline \hline
\end{tabular}

TABLE VII. The partial width ratios for the $n c \bar{c} \bar{c}$ tetraquarks. For each state, we choose one mode as the reference channel, and the partial width ratios of the other channels are calculated relative to this channel. The masses are all in units of $\mathrm{MeV}$.

\begin{tabular}{lcccccc}
\hline \hline System & $J^{P}$ & Mass & $\bar{D}^{*} J / \psi$ & $\bar{D}^{*} \eta_{c}$ & $\bar{D} J / \psi$ & $\bar{D} \eta_{c}$ \\
\hline$n c \bar{c} \bar{c}$ & $0^{+}$ & 4936.7 & $\times$ & & & 1 \\
& & 5185.3 & 6.8 & & & 1 \\
& $1^{+}$ & 4968.1 & $\times$ & $\times$ & 1 & \\
& & 5135.5 & 0.004 & 1.3 & 1 & \\
& & 5154.0 & 20.7 & 1.3 & 1 & \\
& $2^{+}$ & 5198.4 & 1 & & & \\
\hline \hline
\end{tabular}

TABLE VIII. The partial width ratios for the $s c \bar{c} \bar{c}$ tetraquarks. For each state, we choose one mode as the reference channel, and the partial width ratios of the other channels are calculated relative to this channel. The masses are all in units of $\mathrm{MeV}$.

\begin{tabular}{lcccccc}
\hline \hline System & $J^{P}$ & Mass & $\bar{D}_{s}^{*} J / \psi$ & $\bar{D}_{s}^{*} \eta_{c}$ & $\bar{D}_{s} J / \psi$ & $\bar{D}_{s} \eta_{c}$ \\
\hline$s c \bar{c} \bar{c}$ & $0^{+}$ & 5040.1 & $\times$ & & & 1 \\
& & 5290.6 & 7.0 & & & 1 \\
& $1^{+}$ & 5069.2 & $\times$ & $\times$ & 1 & \\
& & 5239.9 & 0.005 & 1.3 & 1 & \\
& & 5254.3 & 22.1 & 1.3 & 1 & \\
& $2^{+}$ & 5303.3 & 1 & & & \\
\hline \hline
\end{tabular}

$$
\frac{\Gamma\left[T\left(n c \bar{c} \bar{c}, 5185.3,0^{+}\right) \rightarrow \bar{D}^{*} J / \psi\right]}{\Gamma\left[T\left(n c \bar{c} \bar{c}, 5185.3,0^{+}\right) \rightarrow \bar{D} J / \psi\right]} \sim 6.8
$$

and

$$
\frac{\Gamma\left[T\left(s c \bar{c} \bar{c}, 5290.6,0^{+}\right) \rightarrow \bar{D}_{s}^{*} J / \psi\right]}{\Gamma\left[T\left(s c \bar{c} \bar{c}, 5290.6,0^{+}\right) \rightarrow \bar{D}_{s} J / \psi\right]} \sim 7.0
$$

The dominant decay modes are the $\bar{D}_{(s)}^{*} J / \psi$ final states. Next we consider the axial-vector states. The $T\left(n c \bar{c} \bar{c}, 5135.3,1^{+}\right)$decay dominantly to $\bar{D}^{*} \eta_{c}$ and $\bar{D} J / \psi$

TABLE IX. Masses and eigenvectors of the $n b \bar{c} \bar{c}$ and $s b \bar{c} \bar{c}$ tetraquarks. The masses are all in units of $\mathrm{MeV}$.

\begin{tabular}{llcc}
\hline \hline System & $J^{P}$ & Mass & Eigenvector \\
\hline$n b \bar{c} \bar{c}$ & $0^{+}$ & 8199.1 & $\{0.907,0.421\}$ \\
& & 8444.8 & $\{-0.421,0.907\}$ \\
& $1^{+}$ & 8217.5 & $\{0.958,-0.151,-0.244\}$ \\
& & 8430.0 & $\{0.207,0.953,0.222\}$ \\
& & 8454.4 & $\{-0.199,0.263,-0.944\}$ \\
& $2^{+}$ & 8477.9 & $\{1\}$ \\
& $0_{\bar{c} \bar{c}}$ & 8300.5 & $\{0.895,0.445\}$ \\
& $0^{+}$ & 8538.7 & $\{-0.445,0.895\}$ \\
& & 8324.8 & $\{0.952,-0.169,-0.256\}$ \\
& $1^{+}$ & 8521.9 & $\{0.211,0.967,0.145\}$ \\
& & 8553.3 & $\{-0.223,0.192,-0.956\}$ \\
& $2^{+}$ & 8569.1 & $\{1\}$ \\
\hline \hline
\end{tabular}




$$
\Gamma_{\bar{D}^{*} J / \psi}: \Gamma_{\bar{D}^{*} \eta_{c}}: \Gamma_{\bar{D} J / \psi} \sim 0.004: 1.3: 1 .
$$

And for $T\left(n c \bar{c} \bar{c}, 5154.0,1^{+}\right)$

$$
\Gamma_{\bar{D}^{*} J / \psi}: \Gamma_{\bar{D}^{*} \eta_{c}}: \Gamma_{\bar{D} J / \psi} \sim 20.7: 1.3: 1 .
$$

Its dominant decay mode is $\bar{D}^{*} J / \psi$. The decay property of the $s c \bar{c} \bar{c}$ tetraquarks is similar the $n c \bar{c} \bar{c}$ tetraquarks.
Replacing the charm quark by a bottom quark, we get the $n b \bar{c} \bar{c}$ and $s b \bar{c} \bar{c}$ tetraquarks. We list their masses and eigenvectors in Table IX and plot their relative position in Fig. 2, along with the possible decay channels. Since the $B_{c}^{*}$ meson has not been observed, we use the GodfreyIsgur (GI) model's prediction $M_{B_{c}^{*}}=6338 \mathrm{MeV}$ [83] to estimate the meson-meson thresholds. The $q b \bar{c} \bar{c}$ tetraquarks are very similar to the $q c \bar{c} \bar{c}$ tetraquarks. The ground states are both scalar. They are $T\left(n b \bar{c} \bar{c}, 8199.1,0^{+}\right)$and

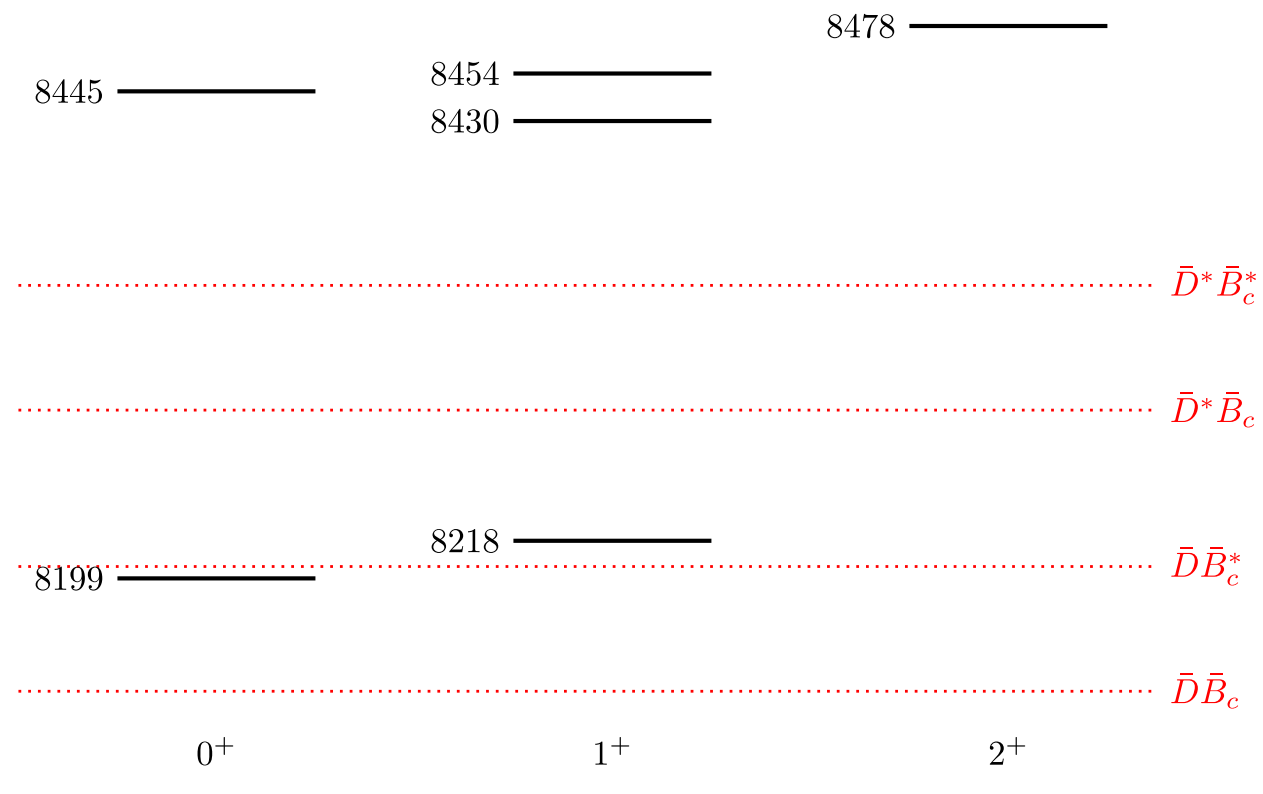

(a) $n b \bar{c} \bar{c}$ states

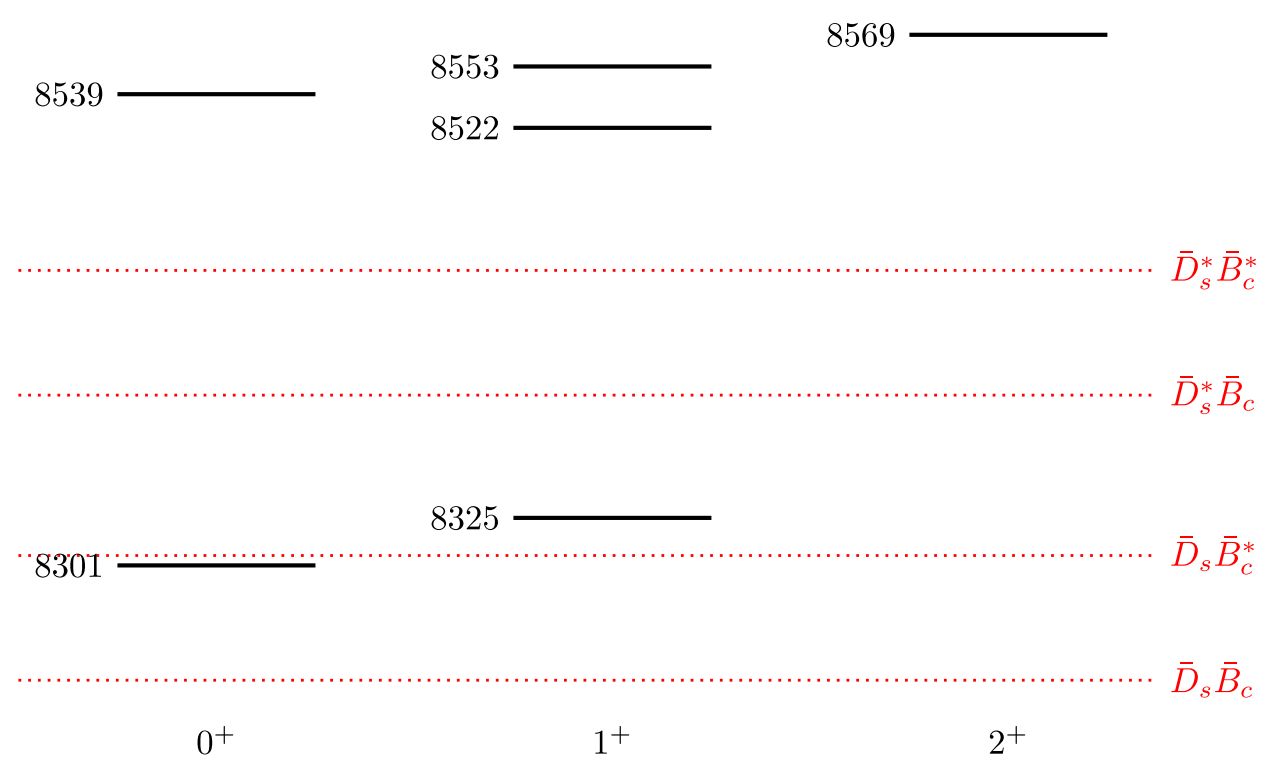

(b) $s b \bar{c} \bar{c}$ states

FIG. 2. Mass spectra of the $n b \bar{c} \bar{c}$ and $s b \bar{c} \bar{c}$ tetraquark states. The dotted lines indicate various meson-meson thresholds. Here the predicted mass $M_{B_{c}^{*}}=6338 \mathrm{MeV}$ of Godfrey et al. [83] is used. The masses are all in units of MeV. 
$T\left(s b \bar{c} \bar{c}, 8300.5,0^{+}\right)$, whose color configurations are $6_{c} \otimes \overline{6}_{c}$ as well $(>80 \%)$. The lightest axial-vector states, $T\left(n b \bar{c} \bar{c}, 8217.5,1^{+}\right)$and $T\left(s b \bar{c} \bar{c}, 8324.8,1^{+}\right)$are also dominated by $6_{c} \otimes \overline{6}_{c}$ component (>90\%). All higher states mainly consist of $\overline{3}_{c} \otimes 3_{c}$ component.

The decay properties of the $q b \bar{c} \bar{c}$ tetraquarks can be found from Tables X-XV. Similar to the $q c \bar{c} \bar{c}$ tetraquarks, the two lowest states are just above their $S$-wave decay

TABLE X. The eigenvectors of the $n b \bar{c} \bar{c}$ tetraquarks in the $n \bar{c} \otimes b \bar{c}$ configuration. The masses are all in units of $\mathrm{MeV}$.

\begin{tabular}{llcrrrr}
\hline \hline System & $J^{P}$ & Mass & $\bar{D}^{*} \bar{B}_{c}^{*}$ & $\bar{D}^{*} \bar{B}_{c}$ & $\bar{D} \bar{B}_{c}^{*}$ & $\bar{D} \bar{B}_{c}$ \\
\hline$n b \bar{c} \bar{c}$ & $0^{+}$ & 8199.1 & 0.520 & & & 0.581 \\
& & 8444.8 & -0.559 & & & 0.282 \\
& $1^{+}$ & 8217.5 & 0.454 & 0.400 & -0.523 & \\
& & 8430.0 & 0.210 & 0.409 & 0.369 & \\
& & 8454.4 & -0.500 & 0.299 & -0.084 & \\
& $2^{+}$ & 8477.9 & 0.577 & & & \\
\hline \hline
\end{tabular}

TABLE XI. The eigenvectors of the $s b \bar{c} \bar{c}$ tetraquarks in the $s \bar{c} \otimes b \bar{c}$ configuration. The masses are all in units of $\mathrm{MeV}$.

\begin{tabular}{lcrrrrr}
\hline \hline System & $J^{P}$ & Mass & $\bar{D}_{s}^{*} \bar{B}_{c}^{*}$ & $\bar{D}_{s}^{*} \bar{B}_{c}$ & $\bar{D}_{s} \bar{B}_{c}^{*}$ & $\bar{D}_{s} \bar{B}_{c}$ \\
\hline$s b \bar{c} \bar{c}$ & $0^{+}$ & 8300.5 & 0.505 & & & 0.588 \\
& & 8538.7 & -0.573 & & & 0.266 \\
& $1^{+}$ & 8324.8 & 0.445 & 0.394 & -0.532 & \\
& & 8521.9 & 0.181 & 0.439 & 0.350 & \\
& & 8553.3 & -0.519 & 0.263 & -0.106 & \\
& $2^{+}$ & 8569.1 & 0.577 & & & \\
\hline \hline
\end{tabular}

TABLE XII. The values of $k \cdot\left|c_{i}\right|^{2}$ for the $n b \bar{c} \bar{c}$ tetraquarks (in units of $\mathrm{MeV}$ ).

\begin{tabular}{lccccrr}
\hline \hline System & $J^{P}$ & Mass & $\bar{D}^{*} \bar{B}_{c}^{*}$ & $\bar{D}^{*} \bar{B}_{c}$ & $\bar{D}^{*} \bar{B}_{c}^{*}$ & $\bar{D} \bar{B}_{c}$ \\
\hline$n b \bar{c} \bar{c}$ & $0^{+}$ & 8199.1 & $\times$ & & & 137.0 \\
& & 8444.8 & 172.6 & & & 75.9 \\
& $1^{+}$ & 8217.5 & $\times$ & $\times$ & 51.7 & \\
& & 8430.0 & 22.4 & 112.9 & 111.6 & \\
& & 8454.4 & 144.5 & 65.2 & 6.1 & \\
& $2^{+}$ & 8477.9 & 213.0 & & & \\
\hline \hline
\end{tabular}

TABLE XIII. The values of $k \cdot\left|c_{i}\right|^{2}$ for the $s b \bar{c} \bar{c}$ tetraquarks (in units of $\mathrm{MeV}$ ).

\begin{tabular}{lccccrr}
\hline \hline System & $J^{P}$ & Mass & $\bar{D}_{s}^{*} \bar{B}_{c}^{*}$ & $\bar{D}_{s}^{*} \bar{B}_{c}$ & $\bar{D}_{s} \bar{B}_{c}^{*}$ & $\bar{D}_{s} \bar{B}_{c}$ \\
\hline$s b \bar{c} \bar{c}$ & $0^{+}$ & 8300.5 & $\times$ & & & 143.8 \\
& & 8538.7 & 175.2 & & & 68.0 \\
& $1^{+}$ & 8324.8 & $\times$ & $\times$ & 66.7 & \\
& & 8521.9 & 15.7 & 126.8 & 100.4 & \\
& & 8553.3 & 155.2 & 50.7 & 9.9 & \\
& $2^{+}$ & 8569.1 & 206.3 & & & \\
\hline \hline
\end{tabular}

TABLE XIV. The partial width ratios for the $n b \bar{c} \bar{c}$ tetraquarks. For each state, we choose one mode as the reference channel, and the partial width ratios of the other channels are calculated relative to this channel. The masses are all in units of $\mathrm{MeV}$.

\begin{tabular}{lcccccc}
\hline \hline System & $J^{P}$ & Mass & $\bar{D}^{*} \bar{B}_{c}^{*}$ & $\bar{D}^{*} \bar{B}_{c}$ & $\bar{D}_{\bar{B}}^{*}$ & $\bar{D} \bar{B}_{c}$ \\
\hline$n b \bar{c} \bar{c}$ & $0^{+}$ & 8199.1 & $\times$ & & & 1 \\
& & 8444.8 & 2.3 & & & 1 \\
& $1^{+}$ & 8217.5 & $\times$ & $\times$ & 1 & \\
& & 8430.0 & 0.2 & 1.01 & 1 & \\
& & 8454.4 & 23.8 & 10.7 & 1 & \\
& $2^{+}$ & 8477.9 & 1 & & & \\
\hline \hline
\end{tabular}

TABLE XV. The partial width ratios for the $s b \bar{c} \bar{c}$ tetraquarks. For each state, we choose one mode as the reference channel, and the partial width ratios of the other channels are calculated relative to this channel. The masses are all in units of $\mathrm{MeV}$.

\begin{tabular}{lcccccc}
\hline \hline System & $J^{P}$ & Mass & $\bar{D}_{s}^{*} \bar{B}_{c}^{*}$ & $\bar{D}_{s}^{*} \bar{B}_{c}$ & $\bar{D}_{s} \bar{B}_{c}^{*}$ & $\bar{D}_{s} \bar{B}_{c}$ \\
\hline$s b \bar{c} \bar{c}$ & $0^{+}$ & 8300.5 & $\times$ & & & 1 \\
& & 8538.7 & 2.6 & & & 1 \\
& $1^{+}$ & 8324.8 & $\times$ & $\times$ & 1 & \\
& & 8521.9 & 0.2 & 1.3 & 1 & \\
& & 8553.3 & 15.7 & 5.1 & 1 & \\
& $2^{+}$ & 8569.1 & 1 & & & \\
\hline \hline
\end{tabular}

channels, while all other states are above all meson-meson thresholds. The higher scalar states can decay into $\bar{D}_{(s)}^{*} \bar{B}_{c}^{*}$ and $\bar{D}_{(s)} \bar{B}_{c}$ channels with into comparable partial widths

$$
\frac{\Gamma\left[T\left(n b \bar{c} \bar{c}, 8444.8,0^{+}\right) \rightarrow \bar{D}^{*} \bar{B}_{c}^{*}\right]}{\Gamma\left[T\left(n b \bar{c} \bar{c}, 8444.8,0^{+}\right) \rightarrow \bar{D}_{c}\right]} \sim 2.3,
$$

and

$$
\frac{\Gamma\left[T\left(s b \bar{c} \bar{c}, 8538.7,0^{+}\right) \rightarrow \bar{D}_{s}^{*} \bar{B}_{c}^{*}\right]}{\Gamma\left[T\left(s b \bar{c} \bar{c}, 8538.7,0^{+}\right) \rightarrow \bar{D}_{s} \bar{B}_{c}\right]} \sim 2.6 .
$$

For the highest axial-vector states, we have

$$
\Gamma_{\bar{D}^{*} \bar{B}_{c}^{*}}: \Gamma_{\bar{D}^{*} \bar{B}_{c}}: \Gamma_{\bar{D} \bar{B}_{c}^{*}} \sim 23.8: 10.7: 1
$$

for $T\left(n b \bar{c} \bar{c}, 8454.5,1^{+}\right)$and

$$
\Gamma_{\bar{D}_{s}^{*} \bar{B}_{c}^{*}}: \Gamma_{\bar{D}_{s}^{*} \bar{B}_{c}}: \Gamma_{\bar{D}_{s} \bar{B}_{c}^{*}} \sim 15.7: 5.1: 1
$$

for $T\left(s b \bar{c} \bar{c}, 8553.3,1^{+}\right)$. The $\bar{D}_{(s)}^{*} \bar{B}_{c}^{*}$ and $\bar{D}_{(s)}^{*} \bar{B}_{c}$ modes are more important. However, for the second higher axialvector states of $q b \bar{c} \bar{c}$ tetraquarks, the partial decay widths of $\bar{D}_{(s)}^{*} \bar{B}_{c}^{*}, \bar{D}_{(s)}^{*} \bar{B}_{c}$ and $\bar{D}_{(s)} \bar{B}_{c}^{*}$ modes are comparable, though the first mode is relatively smaller. 


\section{B. The $q b \bar{b} \bar{b}$ and $q c \bar{b} \bar{b}$ systems}

Next we consider the $q b \bar{b} \bar{b}$ and $q c \bar{b} \bar{b}$ tetraquarks. Their masses and eigenvectors are listed in Tables XVI and XVII. Their relative position are ploted in Figs. 3 and 4. By replacing the three charm quark/antiquarks of the $q c \bar{c} \bar{c}$ tetraquarks into bottom quark/antiquarks, we can obtain the $q b \bar{b} \bar{b}$ tetraquarks. Thus we expect that the two systems share some similar properties. Specifically, the quantum numbers of the lowest and highest $q b \bar{b} \bar{b}$ tetraquarks are $J^{P}=0^{+}$and $J^{P}=2^{+}$respectively. Their ground states are $T\left(n b \bar{b} \bar{b}, 14706.1,0^{+}\right)$and $T\left(s b \bar{b} \bar{b}, 14793.1,0^{+}\right)$, whose dominant color configurations are $6_{c} \otimes \overline{6}_{c}(>85 \%)$. The color triplet component is mostly in the higher scalar states. The axial-vector states are similar. The $6_{c} \otimes \overline{6}_{c}$ component is mostly in the lightest states ( $>90 \%$ ), and the $\overline{3}_{c} \otimes 3_{c}$ components are mostly in the higher states. Just like the $q c \bar{c} \bar{c}$ cases, the underlining reason is the color interaction

$$
\left\langle H_{\mathrm{C}}(q b \bar{b} \bar{b})\right\rangle=m_{q \bar{b}}+m_{b \bar{b}}-\frac{3}{2} \delta m^{\prime}\left\langle V_{12}^{\mathrm{C}}+V_{34}^{\mathrm{C}}\right\rangle
$$

TABLE XVI. Masses and eigenvectors of the $n b \bar{b} \bar{b}$ and $s b \bar{b} \bar{b}$ tetraquarks. The masses are all in units of MeV.

\begin{tabular}{cccc}
\hline \hline System & $J^{P}$ & Mass & Eigenvector \\
\hline$n b \bar{b} \bar{b}$ & $0^{+}$ & 14706.1 & $\{0.932,0.362\}$ \\
& & 14850.9 & $\{-0.362,0.932\}$ \\
& $1^{+}$ & 14712.1 & $\{0.977,0.049,-0.208\}$ \\
& & 14845.1 & $\{-0.097,0.969,-0.228\}$ \\
& & 14851.6 & $\{0.191,0.243,0.951\}$ \\
& $2^{+}$ & 14871.8 & $\{1\}$ \\
$s b \bar{b} \bar{b}$ & $0^{+}$ & 14793.1 & $\{0.924,0.382\}$ \\
& & 14936.2 & $\{-0.382,0.924\}$ \\
& $1^{+}$ & 14804.5 & $\{0.976,0.041,-0.215\}$ \\
& & 14929.2 & $\{0.058,-0.996,0.070\}$ \\
& & 14943.7 & $\{-0.211,-0.081,-0.974\}$ \\
& $2^{+}$ & 14956.6 & $\{1\}$ \\
\hline \hline
\end{tabular}

TABLE XVII. Masses and eigenvectors of the $n c \bar{b} \bar{b}$ and $s c \bar{b} \bar{b}$ tetraquarks. The masses are all in units of $\mathrm{MeV}$.

\begin{tabular}{lccc}
\hline \hline System & $J^{P}$ & Mass & Eigenvector \\
\hline$n c \bar{b} \bar{b}$ & $0^{+}$ & 11581.7 & $\{0.510,0.860\}$ \\
& & 11694.9 & $\{0.860,-0.510\}$ \\
& $1^{+}$ & 11582.2 & $\{-0.425,-0.133,0.895\}$ \\
& & 11624.7 & $\{-0.070,-0.981,-0.179\}$ \\
& & 11658.6 & $\{-0.902,0.139,-0.408\}$ \\
& $2^{+}$ & 11651.7 & $\{1\}$ \\
& $0^{+} \bar{b} \bar{b}$ & 11675.1 & $\{0.545,0.838\}$ \\
& & 11787.2 & $\{0.838,-0.545\}$ \\
& $1^{+}$ & 11673.3 & $\{-0.465,-0.096,0.880\}$ \\
& & 11722.2 & $\{-0.089,-0.984,-0.155\}$ \\
& & 11746.3 & $\{0.881,-0.150,0.449\}$ \\
& $2^{+}$ & 11750.2 & $\{1\}$ \\
\hline \hline
\end{tabular}

where

$$
\delta m^{\prime}=\frac{m_{q b}+m_{b b}-m_{q \bar{b}}-m_{b \bar{b}}}{4} \sim 45 \mathrm{MeV} .
$$

Note that the $\left\langle V_{12}^{\mathrm{C}}+V_{34}^{\mathrm{C}}\right\rangle$ is diagonal in the $\sigma_{c} \otimes \overline{6}_{c}$ and $\overline{3}_{c} \otimes 3_{c}$ color bases, with matrix elements $2 / 3$ and $-4 / 3$ respectively. Thus the $6_{c} \otimes \overline{6}_{c}$ configuration is favored.

The $q c \bar{b} \bar{b}$ tetraquarks turn out to be quite different from the $q b \bar{b} \bar{b}$ tetraquarks. The quantum number of the highest states becomes $J^{P}=0^{+}$. For the $n c \bar{b} \bar{b}$ tetraquarks, the lightest axial-vector state lies only $0.5 \mathrm{MeV}$ above the lightest scalar state. And the ground state of the $s c \bar{b} \bar{b}$ tetraquarks has quantum number $J^{P}=1^{+}$. Moreover, the dominant color configuration of the lower mass states becomes $\overline{3}_{c} \otimes 3_{c}$. For example, the $T\left(n c \bar{b} \bar{b}, 11581.7,0^{+}\right)$ has $74 \%$ of $\overline{3}_{c} \otimes 3_{c}$ component, and the $T(s c \bar{b} \bar{b}$, $\left.11675.1,0^{+}\right)$has $70 \%$. We again resort to the color interaction

$$
\left\langle H_{\mathrm{C}}(q c \bar{b} \bar{b})\right\rangle=m_{q \bar{b}}+m_{c \bar{b}}-\frac{3}{2} \delta m^{\prime \prime}\left\langle V_{12}^{\mathrm{C}}+V_{34}^{\mathrm{C}}\right\rangle
$$

where

$$
\delta m^{\prime \prime}=\frac{m_{q c}+m_{b b}-m_{q \bar{b}}-m_{c \bar{b}}}{4} .
$$

Here $\delta m^{\prime \prime}=-6.5 \mathrm{MeV}$ for $q=n$, and $\delta m^{\prime \prime}=-3.1 \mathrm{MeV}$ for $q=s$. The small negative values indicate that the $\overline{3}_{c} \otimes 3_{c}$ configuration has slightly lower mass. The CM interaction

$$
\begin{aligned}
& \left\langle H_{\mathrm{CM}}(q c \bar{b} \bar{b})\right\rangle \\
& \quad=\left(\begin{array}{cc}
\frac{1}{4}\left(v_{q c}+v_{b b}\right) & -\frac{\sqrt{6}}{4}\left(v_{q \bar{b}}+v_{c \bar{b}}\right) \\
-\frac{\sqrt{6}}{4}\left(v_{q \bar{b}}+v_{c \bar{b}}\right) & \frac{1}{6}\left(v_{q c}+v_{b b}\right)-\frac{1}{3}\left(v_{q \bar{b}}+v_{c \bar{b}}\right)
\end{array}\right)
\end{aligned}
$$

also favors the $\overline{3}_{c} \otimes 3_{c}$ configuration. Thus for both the scalar and the axial-vector states, the lower mass states are dominated by the $\overline{3}_{c} \otimes 3_{c}$ components, while the higher mass states have more $\sigma_{c} \otimes \bar{\sigma}_{c}$ components.

Before concluding this section, we would like to compare the present results with that of Refs. [66,67]. It is interesting to note that the $q b \bar{b} \bar{b}$ tetraquarks (as well as the $q c \bar{c} \bar{c}$ and $q b \bar{c} \bar{c}$ tetraquarks) are very similar to the fully heavy $c b \bar{b} \bar{b}$ tetraquarks, while the $q c \bar{b} \bar{b}$ tetraquarks are more like the doubly heavy tetraquarks. This is quite natural since that the triply heavy tetraquarks are intermediate states between the doubly and fully heavy tetraquarks. The reason is $m_{q} \ll m_{c} \ll m_{b}$. A detailed dynamical study of the dependence of the spectrum and wave function with respect to the quark masses 


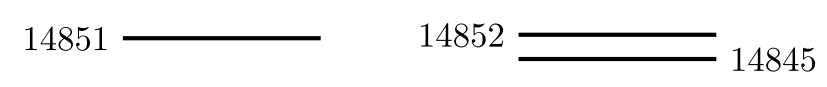

\section{6}

14712

$B \eta_{b}$

$0^{+}$

$1^{+}$

$2^{+}$

(a) $n b \bar{b} \bar{b}$ states

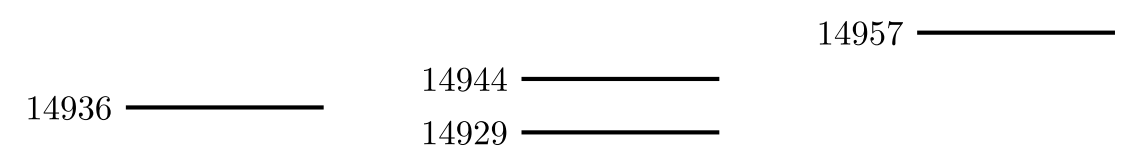

$B_{s}^{*} \Upsilon$

$B_{s} \Upsilon$

$B_{s}^{*} \eta_{b}$

14805

14793
$0^{+}$

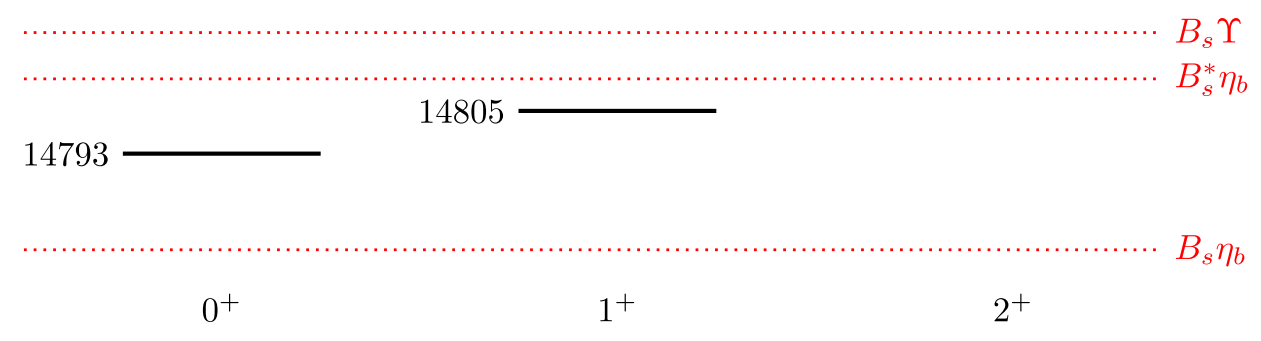

(b) $s b \bar{b} \bar{b}$ states

FIG. 3. Mass spectra of the $n b \bar{b} \bar{b}$ and $s b \bar{b} \bar{b}$ tetraquark states. The dotted lines indicate various meson-meson thresholds. The masses are all in units of $\mathrm{MeV}$.

would be very important for decoding the nature of tetraquarks $[84,85]$.

Next we consider their decay properties. We transform the eigenvectors of the $q b \bar{b} \bar{b}(q c \bar{b} \bar{b})$ tetraquarks into the $q \bar{b} \otimes b \bar{b} \quad(q \bar{b} \otimes c \bar{b}) \quad$ configuration, and calculate the values of $k \cdot\left|c_{i}\right|^{2}$ and partial width ratios of all possible decay channels. The corresponding results are listed in Tables XVIII-XXIX. We find six states which cannot 
11695

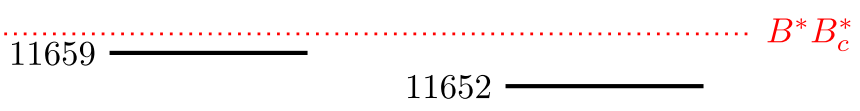

11625

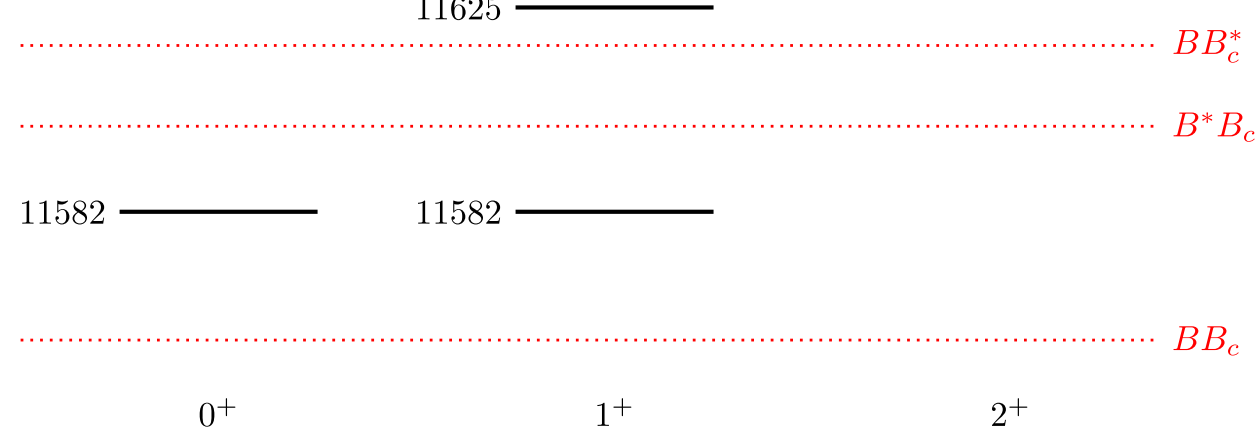

(a) $n c \bar{b} \bar{b}$ states

11787

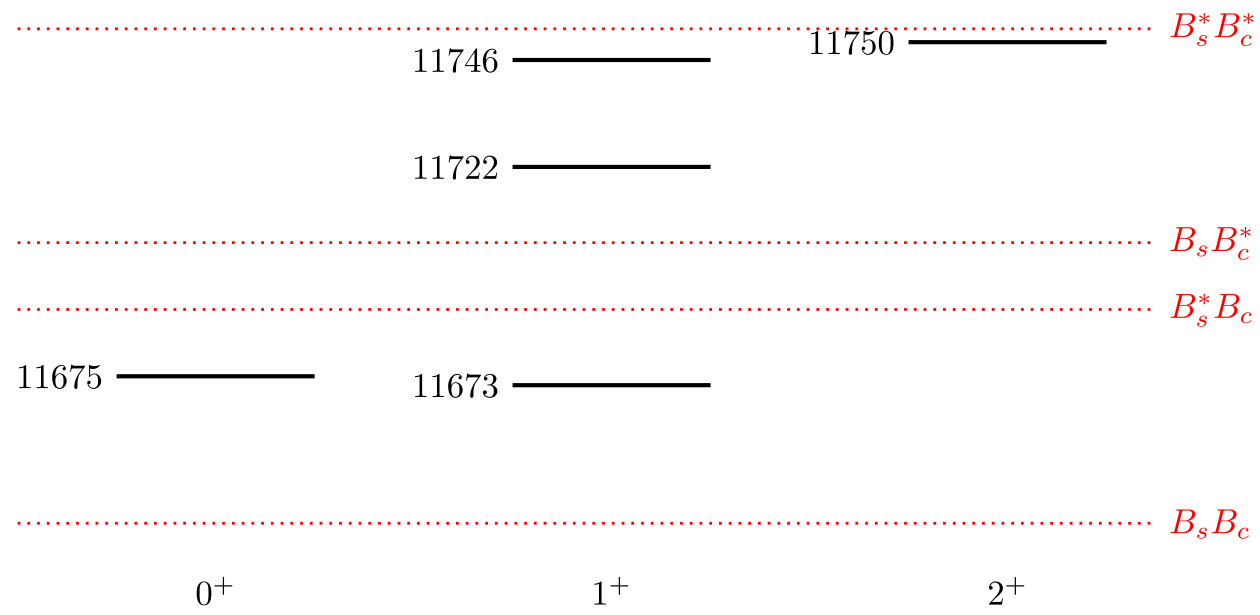

(b) $s c \bar{b} \bar{b}$ states

FIG. 4. Mass spectra of the $n c \bar{b} \bar{b}$ and $s c \bar{b} \bar{b}$ tetraquark states. The dotted lines indicate various meson-meson thresholds. Here the predicted mass $M_{B_{c}^{*}}=6338 \mathrm{MeV}$ of Godfrey et al. [83] is used. The masses are all in units of MeV.

TABLE XVIII. The eigenvectors of the $n b \bar{b} \bar{b}$ tetraquarks in the $n \bar{b} \otimes b \bar{b}$ configuration. The masses are all in units of $\mathrm{MeV}$.

\begin{tabular}{lccrrrr}
\hline \hline System & $J^{P}$ & Mass & \multicolumn{1}{c}{$B^{*} \Upsilon$} & $B^{*} \eta_{b}$ & $B \Upsilon$ & $B \eta_{b}$ \\
\hline$n b \bar{b} \bar{b}$ & $0^{+}$ & 14706.1 & 0.554 & & & 0.562 \\
& & 14850.9 & -0.525 & & & 0.318 \\
& $1^{+}$ & 14712.1 & 0.479 & 0.479 & -0.439 & \\
& & 14845.1 & -0.149 & 0.422 & 0.369 & \\
& & 14851.6 & 0.498 & -0.098 & 0.296 & \\
& $2^{+}$ & 14871.8 & 0.577 & & & \\
\hline \hline
\end{tabular}

TABLE XIX. The eigenvectors of the $s b \bar{b} \bar{b}$ tetraquarks in the $s \bar{b} \otimes b \bar{b}$ configuration. The masses are all in units of $\mathrm{MeV}$.

\begin{tabular}{lcrrrrr}
\hline \hline System & $J^{P}$ & Mass & \multicolumn{1}{c}{$B_{s}^{*} \Upsilon$} & $B_{s}^{*} \eta_{b}$ & $B_{s} \Upsilon$ & $B_{s} \eta_{b}$ \\
\hline$s b \bar{b} \bar{b}$ & $0^{+}$ & 14793.1 & 0.543 & & & 0.568 \\
& & 14936.2 & -0.537 & & & 0.306 \\
& $1^{+}$ & 14804.5 & 0.476 & 0.477 & -0.444 & \\
& & 14929.2 & 0.062 & -0.403 & -0.410 & \\
& & 14943.7 & -0.520 & 0.162 & -0.228 & \\
& $2^{+}$ & 14956.6 & 0.577 & & & \\
\hline \hline
\end{tabular}


TABLE XX. The values of $k \cdot\left|c_{i}\right|^{2}$ for the $n b \bar{b} \bar{b}$ tetraquarks (in units of $\mathrm{MeV}$ ).

\begin{tabular}{lcccccc}
\hline \hline System & $J^{P}$ & Mass & $B^{*} \Upsilon$ & $B^{*} \eta_{b}$ & $B \Upsilon$ & $B \eta_{b}$ \\
\hline$n b \bar{b} \bar{b}$ & $0^{+}$ & 14706.1 & $\times$ & & & 136.4 \\
& & 14850.9 & 185.2 & & & 109.7 \\
& $1^{+}$ & 14712.1 & $\times$ & $\times$ & $\times$ & \\
& & 14845.1 & 14.2 & 162.0 & 115.6 & \\
& & 14851.6 & 167.7 & 8.9 & 76.4 & \\
& $2^{+}$ & 14871.8 & 257.0 & & & \\
\hline \hline
\end{tabular}

TABLE XXI. The values of $k \cdot\left|c_{i}\right|^{2}$ for the $s b \bar{b} \bar{b}$ tetraquarks (in units of $\mathrm{MeV}$ ).

\begin{tabular}{ccccccc}
\hline \hline System & $J^{P}$ & Mass & $B_{s}^{*} \Upsilon$ & $B_{s}^{*} \eta_{b}$ & $B_{s} \Upsilon$ & $B_{s} \eta_{b}$ \\
\hline$s b \bar{b} \bar{b}$ & $0^{+}$ & 14793.1 & $\times$ & & & 139.5 \\
& & 14936.2 & 186.2 & & & 101.6 \\
& $1^{+}$ & 14804.5 & $\times$ & $\times$ & $\times$ & \\
& & 14929.2 & 2.3 & 144.9 & 140.7 & \\
& & 14943.7 & 185.0 & 24.8 & 46.6 & \\
& $2^{+}$ & 14956.5 & 249.2 & & & \\
\hline \hline
\end{tabular}

TABLE XXII. The partial width ratios for the $n b \bar{b} \bar{b}$ tetraquarks. For each state, we choose one mode as the reference channel, and the partial width ratios of the other channels are calculated relative to this channel. The masses are all in units of $\mathrm{MeV}$.

\begin{tabular}{lcccccc}
\hline \hline System & $J^{P}$ & Mass & $B^{*} \Upsilon$ & $B^{*} \eta_{b}$ & $B \Upsilon$ & $B \eta_{b}$ \\
\hline$n b \bar{b} \bar{b}$ & $0^{+}$ & 14706.1 & $\times$ & & & 1 \\
& & 14850.9 & 1.7 & & & 1 \\
& $1^{+}$ & 14712.1 & $\times$ & $\times$ & $\times$ & \\
& & 14845.1 & 0.1 & 1.4 & 1 & \\
& & 14851.6 & 2.2 & 0.1 & 1 & \\
& $2^{+}$ & 14871.8 & 1 & & & \\
\hline \hline
\end{tabular}

TABLE XXIII. The partial width ratios for the $s b \bar{b} \bar{b}$ tetraquarks. For each state, we choose one mode as the reference channel, and the partial width ratios of the other channels are calculated relative to this channel. The masses are all in units of $\mathrm{MeV}$.

\begin{tabular}{lccllll}
\hline \hline System & $J^{P}$ & Mass & $B^{*} \Upsilon$ & $B^{*} \eta_{b}$ & $B \Upsilon$ & $B \eta_{b}$ \\
\hline$s b \bar{b} \bar{b}$ & $0^{+}$ & 14793.1 & $\times$ & & & 1 \\
& & 14936.2 & 1.8 & & & 1 \\
& $1^{+}$ & 14804.5 & $\times$ & $\times$ & $\times$ & \\
& & 14929.2 & 0.02 & 1.03 & 1 & \\
& & 14943.7 & 3.97 & 0.53 & 1 & \\
& $2^{+}$ & 14956.5 & 1 & & & \\
\hline \hline
\end{tabular}

TABLE XXIV. The eigenvectors of the $n c \bar{b} \bar{b}$ tetraquarks in the $n \bar{b} \otimes c \bar{b}$ configuration. The masses are all in units of $\mathrm{MeV}$.

\begin{tabular}{cccrrrr}
\hline \hline System & $J^{P}$ & Mass & $B^{*} B_{c}^{*}$ & $B^{*} B_{c}$ & $B B_{c}^{*}$ & $B B_{c}$ \\
\hline$n c \bar{b} \bar{b}$ & $0^{+}$ & 11581.7 & 0.112 & & & 0.638 \\
& & 11694.9 & 0.756 & & & 0.096 \\
& $1^{+}$ & 11582.2 & 0.120 & -0.486 & 0.378 & \\
& & 11624.7 & -0.114 & -0.378 & -0.424 & \\
& & 11658.6 & -0.688 & -0.194 & 0.307 & \\
& $2^{+}$ & 11651.7 & 0.577 & & & \\
\hline \hline
\end{tabular}

TABLE XXV. The eigenvectors of the $s c \bar{b} \bar{b}$ tetraquarks in the $s \bar{b} \otimes c \bar{b}$ configuration. The masses are all in units of $\mathrm{MeV}$.

\begin{tabular}{lcrrrrr}
\hline \hline System & $J^{P}$ & Mass & $B_{s}^{*} B_{c}^{*}$ & $B_{s}^{*} B_{c}$ & $B_{s} B_{c}^{*}$ & $B_{s} B_{c}$ \\
\hline$s c \bar{b} \bar{b}$ & $0^{+}$ & 11675.1 & 0.143 & & & 0.642 \\
& & 11787.2 & 0.750 & & & 0.070 \\
& $1^{+}$ & 11673.3 & 0.091 & -0.483 & 0.404 & \\
& & 11722.2 & -0.114 & -0.393 & -0.410 & \\
& & 11746.3 & 0.692 & 0.169 & -0.291 & \\
& $2^{+}$ & 11750.2 & 0.577 & & & \\
\hline \hline
\end{tabular}

TABLE XXVI. The values of $k \cdot\left|c_{i}\right|^{2}$ for the $n c \bar{b} \bar{b}$ tetraquarks (in units of $\mathrm{MeV}$ ).

\begin{tabular}{ccccccr}
\hline \hline System & $J^{P}$ & Mass & $B^{*} B_{c}^{*}$ & $B^{*} B_{c}$ & $B B_{c}^{*}$ & $B B_{c}$ \\
\hline$n c \bar{b} \bar{b}$ & $0^{+}$ & 11581.7 & $\times$ & & & 160.9 \\
& & 11694.9 & 246.9 & & & 8.4 \\
& $1^{+}$ & 11582.2 & $\times$ & $\times$ & $\times$ & \\
& & 11624.7 & $\times$ & 54.2 & 36.7 & \\
& & 11658.6 & $\times$ & 21.9 & 46.0 & \\
& $2^{+}$ & 11651.7 & $\times$ & & & \\
\hline \hline
\end{tabular}

TABLE XXVII. The values of $k \cdot\left|c_{i}\right|^{2}$ for the $s c \bar{b} \bar{b}$ tetraquarks (in units of $\mathrm{MeV}$ ).

\begin{tabular}{lcccccr}
\hline \hline System & $J^{P}$ & Mass & $B_{s}^{*} B_{c}^{*}$ & $B_{s}^{*} B_{c}$ & $B_{s} B_{c}^{*}$ & $B_{s} B_{c}$ \\
\hline$s c \bar{b} \bar{b}$ & $0^{+}$ & 11675.1 & $\times$ & & & 180.6 \\
& & 11787.2 & 250.2 & & & 4.5 \\
& $1^{+}$ & 11673.3 & $\times$ & $\times$ & $\times$ & \\
& & 11722.2 & $\times$ & 66.5 & 53.6 & \\
& & 11746.3 & $\times$ & 16.3 & 41.7 & \\
& $2^{+}$ & 11750.2 & $\times$ & & & \\
\hline \hline
\end{tabular}


TABLE XXVIII. The partial width ratios for the $n c \bar{b} \bar{b}$ tetraquarks. For each state, we choose one mode as the reference channel, and the partial width ratios of the other channels are calculated relative to this channel. The masses are all in units of $\mathrm{MeV}$.

\begin{tabular}{ccccccc}
\hline \hline System & $J^{P}$ & Mass & $B^{*} B_{c}^{*}$ & $B^{*} B_{c}$ & $B B_{c}^{*}$ & $B B_{c}$ \\
\hline$n c \bar{b} \bar{b}$ & $0^{+}$ & 11581.7 & $\times$ & & & 1 \\
& & 11694.9 & 29.5 & & & 1 \\
& $1^{+}$ & 11582.2 & $\times$ & $\times$ & $\times$ & \\
& & 11624.7 & $\times$ & 1.5 & 1 & \\
& & 11658.6 & $\times$ & 0.5 & 1 & \\
& $2^{+}$ & 11651.7 & $\times$ & & & \\
\hline \hline
\end{tabular}

TABLE XXIX. The partial width ratios for the $s c \bar{b} \bar{b}$ tetraquarks. For each state, we choose one mode as the reference channel, and the partial width ratios of the other channels are calculated relative to this channel. The masses are all in units of $\mathrm{MeV}$.

\begin{tabular}{ccccccc}
\hline \hline System & $J^{P}$ & Mass & $B_{s}^{*} B_{c}^{*}$ & $B_{s}^{*} B_{c}$ & $B_{s} B_{c}^{*}$ & $B_{s} B_{c}$ \\
\hline$s c \bar{b} \bar{b}$ & $0^{+}$ & 11675.1 & $\times$ & & & 1 \\
& & 11787.2 & 55.7 & & & 1 \\
& $1^{+}$ & 11673.3 & $\times$ & $\times$ & $\times$ & \\
& & 11722.2 & $\times$ & 1.2 & 1 & \\
& & 11746.3 & $\times$ & 0.4 & 1 & \\
& $2^{+}$ & 11750.2 & $\times$ & & & \\
\hline \hline
\end{tabular}

decay into two mesons through $S$-wave. They are $T\left(n b \bar{b} \bar{b}, 14712.1,1^{+}\right), \quad T\left(s b \bar{b} \bar{b}, 14804.5,1^{+}\right), \quad T(n c \bar{b} \bar{b}$, $\left.11582.2,1^{+}\right), T\left(n c \bar{b} \bar{b}, 11651.7,2^{+}\right), T\left(s c \bar{b} \bar{b}, 11673.3,1^{+}\right)$ and $T\left(s c \bar{b} \bar{b}, 11750.2,2^{+}\right)$. Because of the conservation of the angular momentum and parity, their decays into the thresholds below them must be of higher wave ( $D$-wave for example), which are highly suppressed. Thus we expect them to be narrow.

However, we need to bear in mind that our model is an oversimplified one. The dynamical effects are not included explicitly, but rather concealed in the interaction strengths $\left\{m_{i j}, v_{i j}\right\}$. In the present work, we fit them from the conventional mesons and baryons. But they may be altered in the tetraquarks because of their wave function dependence. Nonetheless, even if the masses of these states are pushed upward above their $S$-wave channels, their phase spaces are still relatively small. Thus their decay widths are still smaller compared to those of other states. A dynamical calculation may help settle this issue. We hope the future experiments can search for these states.

\section{The $q c \bar{c} \bar{b}$ and $q b \bar{c} \bar{b}$ systems}

Now we turn to the systems with two different heavy antiquarks. They are not constrained by Pauli principle. The mass spectra and eigenvectors are listed in Tables XXX-XXXI. We also transform their eigenvectors

TABLE XXX. Masses and eigenvectors of the $n c \bar{c} \bar{b}$ and $s c \bar{c} \bar{b}$ tetraquarks. The masses are all in units of MeV.

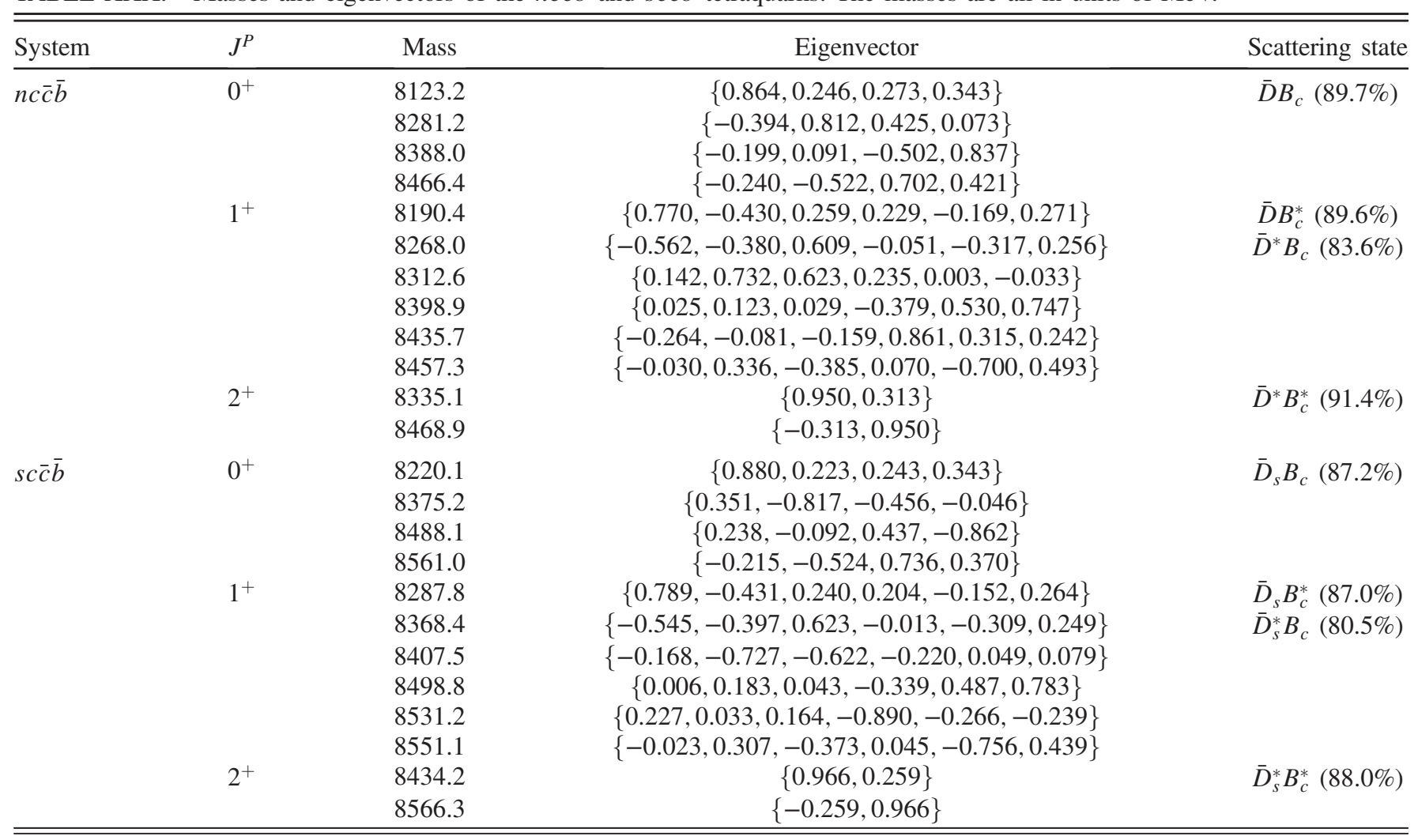


TABLE XXXI. Masses and eigenvectors of the $n b \bar{c} \bar{b}$ and $s b \bar{c} \bar{b}$ tetraquarks. The masses are all in units of MeV.

\begin{tabular}{|c|c|c|c|c|}
\hline System & $J^{P}$ & Mass & Eigenvector & Scattering state \\
\hline \multirow[t]{12}{*}{$n b \bar{c} \bar{b}$} & \multirow[t]{4}{*}{$0^{+}$} & 11253.4 & $\{0.816,0.365,0.364,0.261\}$ & \multirow{4}{*}{$\begin{array}{l}\bar{D} \eta_{b}(96.7 \%) \\
\bar{D}^{*} \Upsilon(86.7 \%)\end{array}$} \\
\hline & & 11438.6 & $\{0.463,-0.835,0.011,-0.296\}$ & \\
\hline & & 11673.1 & $\{0.160,-0.165,-0.688,0.688\}$ & \\
\hline & & 11748.7 & $\{0.306,0.377,-0.628,-0.609\}$ & \\
\hline & \multirow[t]{6}{*}{$1^{+}$} & 11314.8 & $\{0.702,-0.408,0.397,0.298,-0.215,0.219\}$ & \multirow{6}{*}{$\begin{array}{c}\bar{D} \Upsilon(96.2 \%) \\
\bar{D}^{*} \eta_{b}(94.6 \%) \\
\bar{D}^{*} \Upsilon(91.0 \%)\end{array}$} \\
\hline & & 11392.4 & $\{-0.609,-0.450,0.515,-0.218,-0.240,0.239\}$ & \\
\hline & & 11446.6 & $\{-0.024,-0.688,-0.655,-0.028,-0.231,-0.205\}$ & \\
\hline & & 11697.5 & $\{0.080,-0.232,-0.184,-0.236,0.675,0.628\}$ & \\
\hline & & 11719.1 & $\{0.353,0.101,0.028,-0.889,-0.267,-0.047\}$ & \\
\hline & & 11744.9 & $\{-0.070,0.305,-0.337,0.130,-0.562,0.675\}$ & \\
\hline & \multirow[t]{2}{*}{$2^{+}$} & 11453.9 & $\{0.926,0.378\}$ & \multirow[t]{2}{*}{$\bar{D}^{*} \Upsilon(94.9 \%)$} \\
\hline & & 11747.6 & $\{-0.378,0.926\}$ & \\
\hline \multirow[t]{12}{*}{$s b \bar{c} \bar{b}$} & \multirow[t]{4}{*}{$0^{+}$} & 11355.0 & $\{0.816,0.366,0.364,0.262\}$ & \multirow{4}{*}{$\begin{array}{l}\bar{D}_{s} \eta_{b}(96.7 \%) \\
\bar{D}_{s}^{*} \Upsilon(84.7 \%)\end{array}$} \\
\hline & & 11539.4 & $\{0.468,-0.840,-0.008,-0.275\}$ & \\
\hline & & 11760.7 & $\{0.159,-0.124,-0.714,0.670\}$ & \\
\hline & & 11836.7 & $\{0.300,0.381,-0.598,-0.638\}$ & \\
\hline & \multirow[t]{6}{*}{$1^{+}$} & 11416.4 & $\{0.698,-0.405,0.407,0.296,-0.219,0.218\}$ & \multirow{6}{*}{$\begin{array}{l}\bar{D}_{s} \Upsilon(96.4 \%) \\
\bar{D}_{s}^{*} \eta_{b}(94.3 \%) \\
\bar{D}_{s}^{*} \Upsilon(89.9 \%)\end{array}$} \\
\hline & & 11496.1 & $\{-0.615,-0.437,0.522,-0.216,-0.238,0.235\}$ & \\
\hline & & 11549.1 & $\{-0.029,-0.698,-0.651,-0.027,-0.229,-0.185\}$ & \\
\hline & & 11785.1 & $\{0.071,-0.248,-0.151,-0.206,0.717,0.595\}$ & \\
\hline & & 11802.8 & $\{-0.354,-0.087,-0.019,0.899,0.239,0.024\}$ & \\
\hline & & 11833.6 & $\{-0.066,0.296,-0.339,0.115,-0.520,0.713\}$ & \\
\hline & \multirow[t]{2}{*}{$2^{+}$} & 11558.4 & $\{0.926,0.378\}$ & \multirow[t]{2}{*}{$\bar{D}_{s}^{*} \Upsilon(94.9 \%)$} \\
\hline & & 11832.7 & $\{-0.378,0.926\}$ & \\
\hline
\end{tabular}

TABLE XXXII. The eigenvectors of the $n c \bar{c} \bar{b}$ tetraquarks. The masses are all in units of MeV.

\begin{tabular}{|c|c|c|c|c|c|c|c|c|c|c|}
\hline \multirow[b]{2}{*}{ System } & \multirow[b]{2}{*}{$J^{P}$} & \multirow[b]{2}{*}{ Mass } & \multicolumn{4}{|c|}{$n \bar{c} \otimes c \bar{b}$} & \multicolumn{4}{|c|}{$n \bar{b} \otimes c \bar{c}$} \\
\hline & & & $\bar{D}^{*} B_{c}^{*}$ & $\bar{D}^{*} B_{c}$ & $\bar{D} B_{c}^{*}$ & $\overline{\bar{D} B_{c}}$ & $B^{*} J / \psi$ & $B^{*} \eta_{c}$ & $B J / \psi$ & $B \eta_{c}$ \\
\hline \multirow[t]{12}{*}{$\overline{n c \bar{c} \bar{b}}$} & $0^{+}$ & 8123.2 & -0.086 & & & 0.947 & 0.277 & & & 0.473 \\
\hline & & 8281.2 & 0.649 & & & 0.286 & -0.254 & & & -0.801 \\
\hline & & 8388.0 & 0.709 & & & -0.113 & 0.291 & & & 0.314 \\
\hline & & 8466.4 & -0.263 & & & 0.090 & 0.880 & & & -0.187 \\
\hline & $1^{+}$ & 8190.4 & -0.057 & 0.129 & 0.947 & & 0.219 & 0.451 & 0.252 & \\
\hline & & 8268.0 & 0.108 & -0.915 & 0.224 & & 0.337 & -0.415 & -0.192 & \\
\hline & & 8312.6 & 0.770 & 0.233 & 0.123 & & -0.048 & -0.576 & 0.547 & \\
\hline & & 8398.9 & 0.610 & -0.165 & -0.116 & & -0.143 & 0.476 & -0.137 & \\
\hline & & 8435.7 & 0.089 & 0.252 & 0.146 & & -0.015 & -0.245 & -0.762 & \\
\hline & & 8457.3 & -0.113 & -0.039 & 0.061 & & -0.903 & -0.086 & -0.006 & \\
\hline & $2^{+}$ & 8335.1 & 0.956 & & & & 0.595 & & & \\
\hline & & 8468.9 & 0.293 & & & & -0.804 & & & \\
\hline
\end{tabular}


TABLE XXXIII. The eigenvectors of the $s c \bar{c} \bar{b}$ tetraquarks. The masses are all in units of MeV.

\begin{tabular}{|c|c|c|c|c|c|c|c|c|c|c|}
\hline \multirow[b]{2}{*}{ System } & \multirow[b]{2}{*}{$J^{P}$} & \multirow[b]{2}{*}{ Mass } & \multicolumn{4}{|c|}{$s \bar{c} \otimes c \bar{b}$} & \multicolumn{4}{|c|}{$s \bar{b} \otimes c \bar{c}$} \\
\hline & & & $\bar{D}_{s}^{*} B_{c}^{*}$ & $\bar{D}_{s}^{*} B_{c}$ & $\bar{D}_{s} B_{c}^{*}$ & $\bar{D}_{s} B_{c}$ & $B_{s}^{*} J / \psi$ & $B_{s}^{*} \eta_{c}$ & $B_{s} J / \psi$ & $B_{s} \eta_{c}$ \\
\hline \multirow[t]{12}{*}{$\overline{s c \bar{c} \bar{b}}$} & $0^{+}$ & 8220.1 & -0.100 & & & 0.934 & -0.276 & & & 0.508 \\
\hline & & 8375.2 & -0.612 & & & -0.326 & 0.279 & & & 0.796 \\
\hline & & 8488.1 & -0.720 & & & 0.100 & -0.337 & & & -0.261 \\
\hline & & 8561.0 & -0.311 & & & 0.109 & 0.856 & & & -0.199 \\
\hline & $1^{+}$ & 8287.8 & -0.064 & 0.145 & 0.933 & & 0.217 & 0.483 & 0.262 & \\
\hline & & 8368.4 & 0.106 & -0.897 & 0.257 & & 0.361 & -0.419 & -0.200 & \\
\hline & & 8407.5 & -0.726 & -0.238 & -0.135 & & 0.049 & 0.581 & -0.595 & \\
\hline & & 8498.8 & 0.649 & -0.163 & -0.107 & & -0.201 & 0.416 & -0.133 & \\
\hline & & 8531.2 & -0.092 & -0.294 & -0.171 & & 0.065 & 0.268 & 0.721 & \\
\hline & & 8551.1 & -0.168 & -0.062 & 0.072 & & -0.880 & -0.096 & 0.033 & \\
\hline & $2^{+}$ & 8434.2 & 0.938 & & & & 0.639 & & & \\
\hline & & 8566.3 & 0.347 & & & & -0.769 & & & \\
\hline
\end{tabular}

TABLE XXXIV. The eigenvectors of the $n b \bar{c} \bar{b}$ tetraquarks. The masses are all in units of MeV.

\begin{tabular}{|c|c|c|c|c|c|c|c|c|c|c|}
\hline \multirow[b]{2}{*}{ System } & \multirow[b]{2}{*}{$J^{P}$} & \multirow[b]{2}{*}{ Mass } & \multicolumn{4}{|c|}{$n \bar{c} \otimes b \bar{b}$} & \multicolumn{4}{|c|}{$n \bar{b} \otimes b \bar{c}$} \\
\hline & & & $\bar{D}^{*} \Upsilon$ & $\bar{D}^{*} \eta_{b}$ & $\bar{D} \Upsilon$ & $\bar{D} \eta_{b}$ & $B^{*} \bar{B}_{c}^{*}$ & $B^{*} \bar{B}_{c}$ & $B \bar{B}_{c}^{*}$ & $B \bar{B}_{c}$ \\
\hline \multirow[t]{12}{*}{$\overline{n b \bar{c} \bar{b}}$} & $0^{+}$ & 11253.4 & -0.050 & & & 0.983 & -0.356 & & & 0.322 \\
\hline & & 11438.6 & -0.931 & & & -0.094 & 0.257 & & & 0.577 \\
\hline & & 11673.1 & 0.361 & & & -0.099 & 0.197 & & & 0.723 \\
\hline & & 11748.7 & 0.019 & & & -0.119 & -0.877 & & & 0.201 \\
\hline & $1^{+}$ & 11314.8 & -0.005 & 0.072 & 0.981 & & 0.288 & 0.289 & 0.278 & \\
\hline & & 11392.4 & 0.037 & -0.972 & 0.091 & & 0.361 & -0.289 & -0.236 & \\
\hline & & 11446.6 & -0.954 & -0.047 & -0.005 & & 0.008 & 0.420 & -0.425 & \\
\hline & & 11697.5 & 0.292 & -0.056 & -0.044 & & 0.047 & 0.689 & -0.403 & \\
\hline & & 11719.1 & -0.054 & -0.192 & -0.126 & & -0.133 & 0.424 & 0.710 & \\
\hline & & 11744.9 & 0.028 & -0.083 & 0.108 & & -0.876 & -0.048 & -0.139 & \\
\hline & $2^{+}$ & 11453.9 & 0.974 & & & & 0.538 & & & \\
\hline & & 11747.6 & 0.226 & & & & -0.843 & & & \\
\hline
\end{tabular}

TABLE XXXV. The eigenvectors of the $s b \bar{c} \bar{b}$ tetraquarks. The masses are all in units of MeV.

\begin{tabular}{|c|c|c|c|c|c|c|c|c|c|c|}
\hline \multirow[b]{2}{*}{ System } & \multirow[b]{2}{*}{$J^{P}$} & \multirow[b]{2}{*}{ Mass } & \multicolumn{4}{|c|}{$s \bar{c} \otimes b \bar{b}$} & \multicolumn{4}{|c|}{$s \bar{b} \otimes b \bar{c}$} \\
\hline & & & $\bar{D}_{s}^{*} \Upsilon$ & $\bar{D}_{s}^{*} \eta_{b}$ & $\bar{D}_{s} \Upsilon$ & $\bar{D}_{s} \eta_{b}$ & $B_{s}^{*} \bar{B}_{c}^{*}$ & $B_{s}^{*} \bar{B}_{c}$ & $B_{s} \bar{B}_{c}^{*}$ & $B_{s} \bar{B}_{c}$ \\
\hline \multirow[t]{12}{*}{$\overline{s b \bar{c} \bar{b}}$} & $0^{+}$ & 11355.0 & -0.048 & & & 0.984 & -0.356 & & & 0.321 \\
\hline & & 11539.4 & -0.920 & & & -0.095 & 0.263 & & & 0.598 \\
\hline & & 11760.7 & 0.389 & & & -0.102 & 0.152 & & & 0.714 \\
\hline & & 11836.7 & 0.001 & & & -0.115 & -0.884 & & & 0.171 \\
\hline & $1^{+}$ & 11416.4 & 0.001 & 0.066 & 0.982 & & 0.290 & 0.281 & 0.283 & \\
\hline & & 11496.1 & 0.048 & -0.971 & 0.085 & & 0.361 & -0.302 & -0.231 & \\
\hline & & 11549.1 & -0.948 & -0.060 & 0.005 & & 0.009 & 0.426 & -0.437 & \\
\hline & & 11785.1 & 0.306 & -0.048 & -0.039 & & 0.106 & 0.666 & -0.417 & \\
\hline & & 11802.8 & 0.046 & 0.197 & 0.129 & & 0.127 & -0.452 & -0.691 & \\
\hline & & 11833.6 & 0.054 & -0.088 & 0.106 & & -0.871 & -0.012 & -0.158 & \\
\hline & $2^{+}$ & 11558.4 & 0.974 & & & & 0.538 & & & \\
\hline & & 11832.7 & 0.226 & & & & -0.843 & & & \\
\hline
\end{tabular}




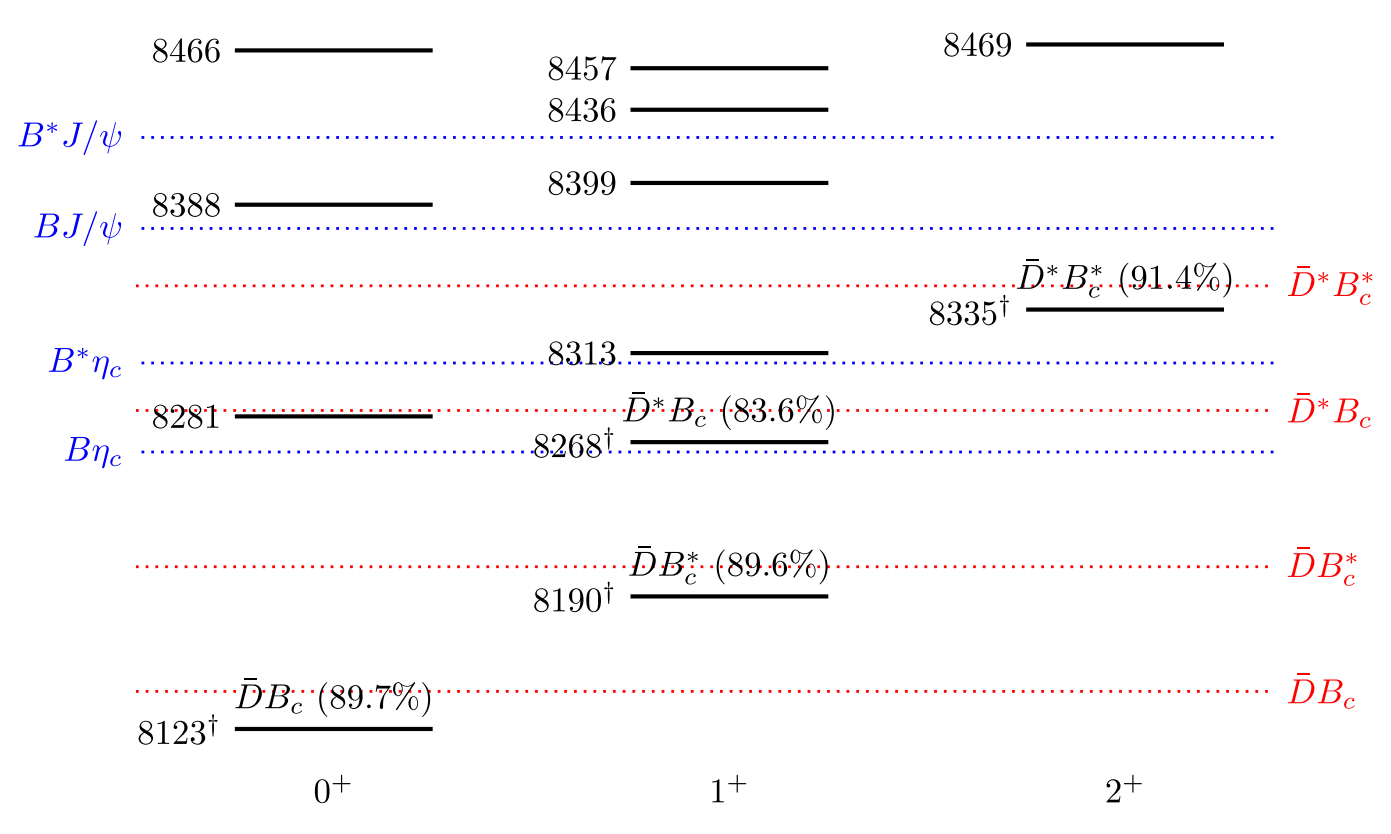

(a) $n c \bar{c} \bar{b}$ states

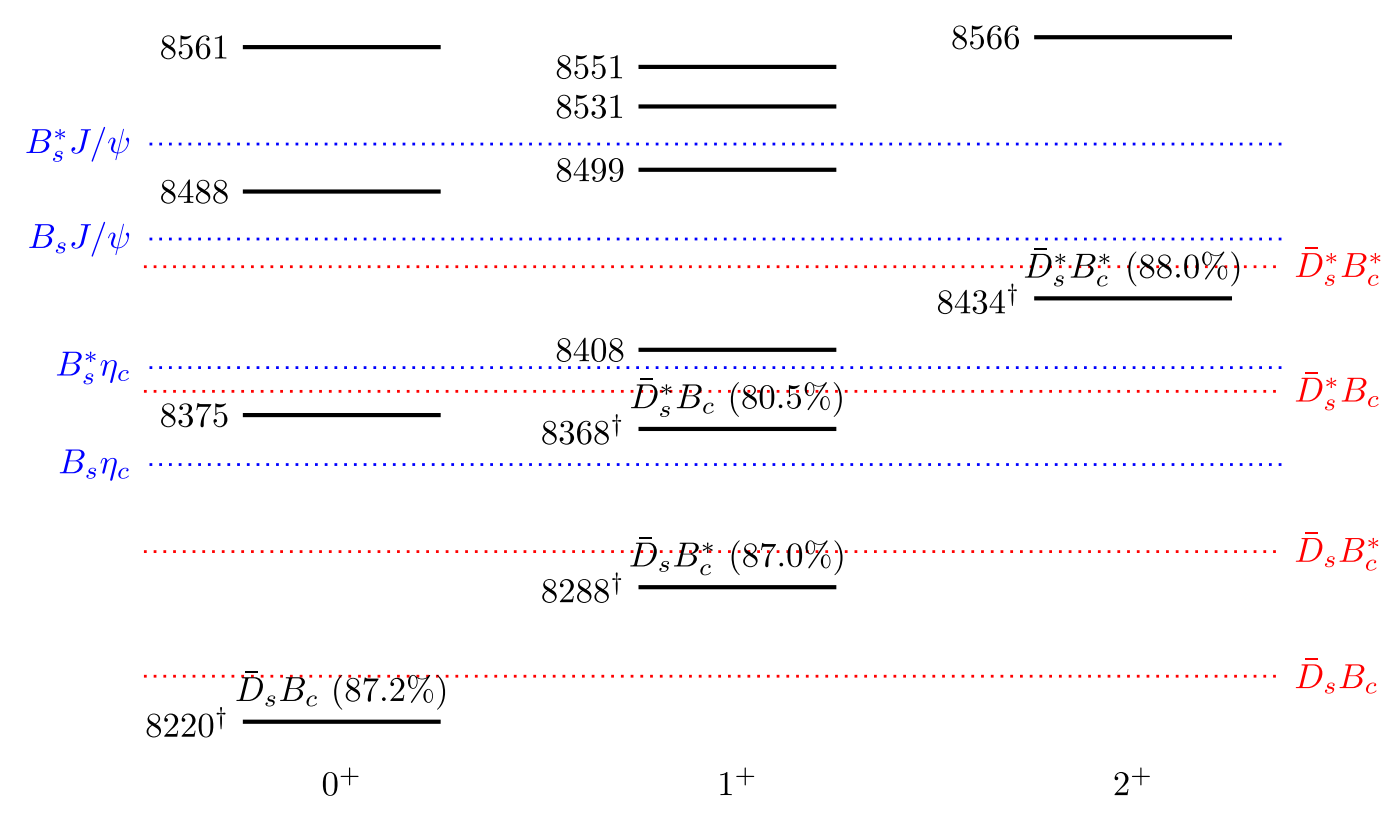

(b) $s c \bar{c} \bar{b}$ states

FIG. 5. Mass spectra of the $n c \bar{c} \bar{b}$ and $s c \bar{c} \bar{b}$ tetraquark states. The dotted lines indicate various meson-meson thresholds. The scattering states are marked with a dagger $(\dagger)$, along with the proportion of their dominant components. Here the predicted mass $M_{B_{c}^{*}}=$ $6338 \mathrm{MeV}$ of Godfrey et al. [83] is used. The masses are all in units of MeV. 


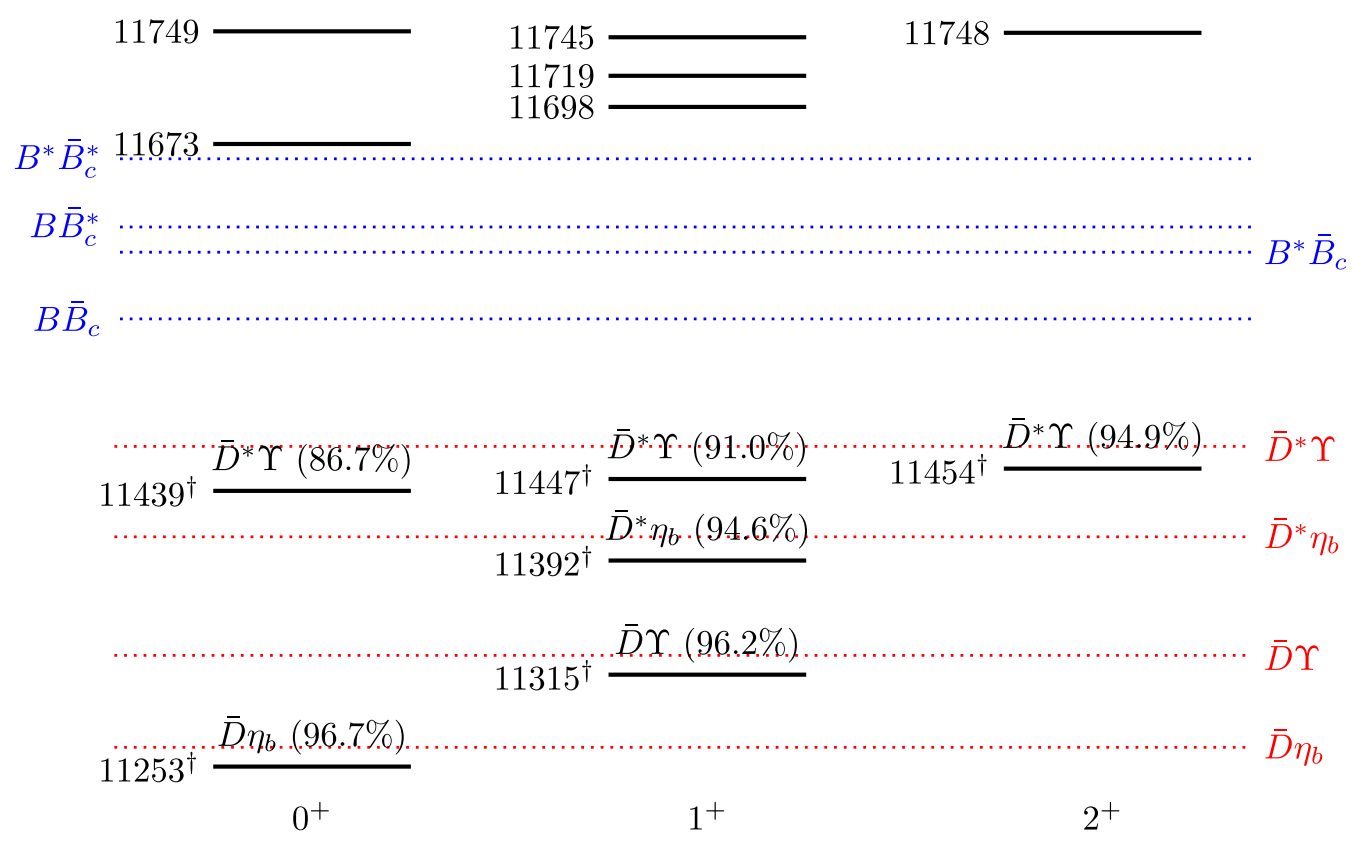

(a) $n b \bar{c} \bar{b}$ states

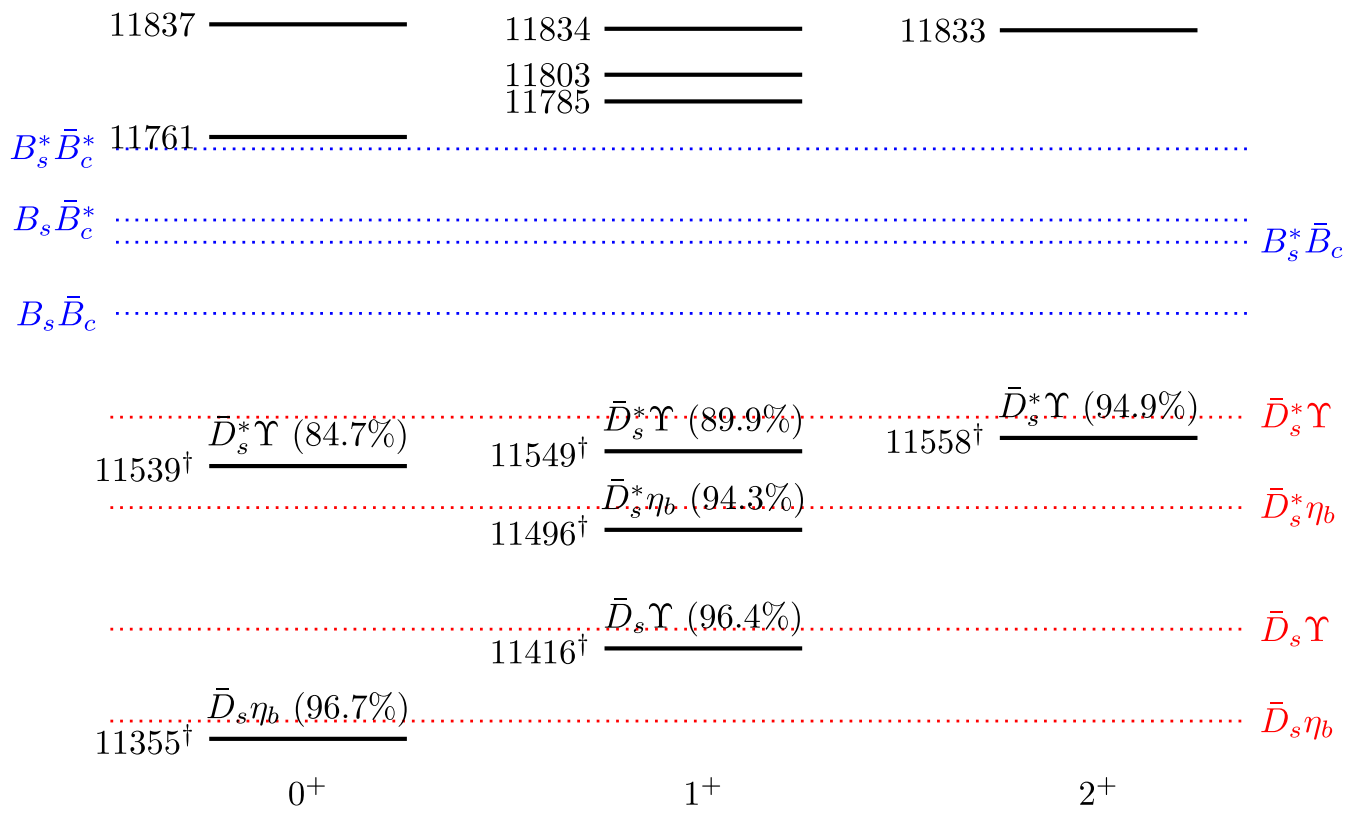

(b) $s b \bar{c} \bar{b}$ states

FIG. 6. Mass spectra of the $n b \bar{c} \bar{b}$ and $s b \bar{c} \bar{b}$ tetraquark states. The dotted lines indicate various meson-meson thresholds. The scattering states are marked with a dagger $(\dagger)$, along with the proportion of their dominant components. Here the predicted mass $M_{B_{c}^{*}}=$ $6338 \mathrm{MeV}$ of Godfrey et al. [83] is used. The masses are all in units of MeV. 
TABLE XXXVI. The values of $k \cdot\left|c_{i}\right|^{2}$ for the $n c \bar{c} \bar{b}$ tetraquarks (in units of MeV).

\begin{tabular}{|c|c|c|c|c|c|c|c|c|c|c|}
\hline \multirow[b]{2}{*}{ System } & \multirow[b]{2}{*}{$J^{P}$} & \multirow[b]{2}{*}{ Mass } & \multicolumn{4}{|c|}{$n \bar{c} \otimes c \bar{b}$} & \multicolumn{4}{|c|}{$n \bar{b} \otimes c \bar{c}$} \\
\hline & & & $\bar{D}^{*} B_{c}^{*}$ & $\bar{D}^{*} B_{c}$ & $\bar{D} B_{c}^{*}$ & $\bar{D} B_{c}$ & $B^{*} J / \psi$ & $B^{*} \eta_{c}$ & $B J / \psi$ & $B \eta_{c}$ \\
\hline \multirow[t]{12}{*}{$\overline{n c \bar{c} \bar{b}}$} & $0^{+}$ & 8123.2 & $x$ & & & $x$ & $x$ & & & $x$ \\
\hline & & 8281.2 & $x$ & & & 52.3 & $x$ & & & 170.0 \\
\hline & & 8388.0 & 179.3 & & & 10.9 & $\times$ & & & 68.6 \\
\hline & & 8466.4 & 42.3 & & & 8.0 & 325.1 & & & 31.0 \\
\hline & $1^{+}$ & 8190.4 & $x$ & $x$ & $x$ & & $x$ & $x$ & $x$ & \\
\hline & & 8268.0 & $x$ & $x$ & 21.5 & & $x$ & $x$ & $x$ & \\
\hline & & 8312.6 & $\times$ & 16.1 & 8.5 & & $x$ & 43.5 & $x$ & \\
\hline & & 8398.9 & 149.1 & 16.2 & 10.3 & & $\times$ & 133.8 & 5.6 & \\
\hline & & 8435.7 & 4.1 & 43.7 & 17.8 & & 0.1 & 42.1 & 280.3 & \\
\hline & & 8457.3 & 7.5 & 1.1 & 3.2 & & 305.7 & 5.6 & 0.02 & \\
\hline & $2^{+}$ & 8335.1 & $x$ & & & & $x$ & & & \\
\hline & & 8468.9 & 52.8 & & & & 278.7 & & & \\
\hline
\end{tabular}

TABLE XXXVII. The values of $k \cdot\left|c_{i}\right|^{2}$ for the $s c \bar{c} \bar{b}$ tetraquarks in the $s \bar{c} \otimes c \bar{b}$ configuration (in units of MeV).

\begin{tabular}{|c|c|c|c|c|c|c|c|c|c|c|}
\hline \multirow[b]{2}{*}{ System } & \multirow[b]{2}{*}{$J^{P}$} & \multirow[b]{2}{*}{ Mass } & \multicolumn{4}{|c|}{$s \bar{c} \otimes c \bar{b}$} & \multicolumn{4}{|c|}{$s \bar{b} \otimes c \bar{c}$} \\
\hline & & & $\bar{D}_{s}^{*} B_{c}^{*}$ & $\bar{D}_{s}^{*} B_{c}$ & $\bar{D}_{s} B_{c}^{*}$ & $\bar{D}_{s} B_{c}$ & $B_{s}^{*} J / \psi$ & $B_{s}^{*} \eta_{c}$ & $B_{s} J / \psi$ & $B_{s} \eta_{c}$ \\
\hline \multirow[t]{12}{*}{$s c \bar{c} \bar{b}$} & $0^{+}$ & 8220.1 & $x$ & & & $x$ & $x$ & & & $x$ \\
\hline & & 8375.2 & $\times$ & & & 67.5 & $x$ & & & 196.4 \\
\hline & & 8488.1 & 180.3 & & & 8.8 & $x$ & & & 49.8 \\
\hline & & 8561.0 & 57.6 & & & 11.9 & 321.4 & & & 35.9 \\
\hline & $1^{+}$ & 8287.8 & $\times$ & $x$ & $x$ & & $\times$ & $x$ & $x$ & \\
\hline & & 8368.4 & $\times$ & $x$ & 28.7 & & $x$ & $x$ & $x$ & \\
\hline & & 8407.5 & $x$ & 14.4 & 10.2 & & $x$ & 61.9 & $x$ & \\
\hline & & 8498.8 & 165.9 & 16.0 & 8.8 & & $x$ & 107.8 & 6.5 & \\
\hline & & 8531.2 & 4.3 & 58.9 & 24.5 & & 1.2 & 51.7 & 268.0 & \\
\hline & & 8551.1 & 16.0 & 2.8 & 4.6 & & 303.7 & 7.1 & 0.7 & \\
\hline & $2^{+}$ & 8434.2 & $\times$ & & & & $\times$ & & & \\
\hline & & 8566.3 & 73.5 & & & & 273.3 & & & \\
\hline
\end{tabular}

TABLE XXXVIII. The partial width ratios for the $n c \bar{c} \bar{b}$ tetraquarks decay into $n \bar{c} \otimes c \bar{b}$ modes. For each state, we choose one mode as the reference channel, and the partial width ratios of the other channels are calculated relative to this channel. The masses are all in units of $\mathrm{MeV}$.

\begin{tabular}{ccccccc}
\hline \hline System & $J^{P}$ & Mass & $\bar{D}^{*} B_{c}^{*}$ & $\bar{D}^{*} B_{c}$ & $\bar{D} B_{c}^{*}$ & $\bar{D} B_{c}$ \\
\hline$n c \bar{c} \bar{b}$ & $0^{+}$ & 8123.2 & $\times$ & & & $\times$ \\
& & 8281.2 & $\times$ & & & 1 \\
& & 8388.0 & 16.4 & & & 1 \\
& & 8466.4 & 5.3 & & & 1 \\
& $1^{+}$ & 8190.4 & $\times$ & $\times$ & $\times$ & \\
& & 8268.0 & $\times$ & $\times$ & 1 & \\
& & 8312.6 & $\times$ & 1.9 & 1 & \\
& & 8398.9 & 14.5 & 1.6 & 1 & \\
& & 8435.7 & 0.2 & 2.5 & 1 & \\
& & 8457.3 & 2.3 & 0.3 & 1 & \\
& 2335.1 & $\times$ & & & \\
& & 8468.9 & 1 & & & \\
\hline \hline
\end{tabular}

TABLE XXXIX. The partial width ratios for the $n c \bar{c} \bar{b}$ tetraquarks decay into $n \bar{b} \otimes c \bar{c}$ modes. For each state, we choose one mode as the reference channel, and the partial width ratios of the other channels are calculated relative to this channel. The masses are all in units of $\mathrm{MeV}$.

\begin{tabular}{ccccccc}
\hline \hline System & $J^{P}$ & Mass & $B^{*} J / \psi$ & $B^{*} \eta_{c}$ & $B J / \psi$ & $B \eta_{c}$ \\
\hline$n c \bar{c} \bar{b}$ & $0^{+}$ & 8123.2 & $\times$ & & & $\times$ \\
& & 8281.2 & $\times$ & & & 1 \\
& & 8388.0 & $\times$ & & & 1 \\
& & 8466.4 & 10.5 & & & 1 \\
& $1^{+}$ & 8190.4 & $\times$ & $\times$ & $\times$ & \\
& & 8268.0 & $\times$ & $\times$ & $\times$ & \\
& & 8312.6 & $\times$ & 1 & $\times$ & \\
& & 8398.9 & $\times$ & 1 & 0.04 & \\
& & 8435.7 & 0.001 & 1 & 6.7 & \\
& & 8457.3 & 54.3 & 1 & 0.003 & \\
& & 8335.1 & $\times$ & & & \\
& 8468.9 & 1 & & & \\
\hline \hline
\end{tabular}


TABLE XL. The partial width ratios for the $s c \bar{c} \bar{b}$ tetraquarks decay into $s \bar{c} \otimes c \bar{b}$ modes. For each state, we choose one mode as the reference channel, and the partial width ratios of the other channels are calculated relative to this channel. The masses are all in units of $\mathrm{MeV}$.

\begin{tabular}{ccccccc}
\hline \hline System & $J^{P}$ & Mass & $\bar{D}_{s}^{*} B_{c}^{*}$ & $\bar{D}_{s}^{*} B_{c}$ & $\bar{D}_{s} B_{c}^{*}$ & $\bar{D}_{s} B_{c}$ \\
\hline$s c \bar{c} \bar{b}$ & $0^{+}$ & 8220.1 & $\times$ & & & $\times$ \\
& & 8375.2 & $\times$ & & & 1 \\
& & 8488.1 & 20.5 & & & 1 \\
& & 8561.0 & 4.8 & & & 1 \\
& $1^{+}$ & 8287.8 & $\times$ & $\times$ & $\times$ & \\
& & 8368.4 & $\times$ & $\times$ & 1 & \\
& & 8407.5 & $\times$ & 1.4 & 1 & \\
& & 8498.8 & 18.9 & 1.8 & 1 & \\
& & 8531.2 & 0.2 & 2.4 & 1 & \\
& 2551.1 & 3.5 & 0.6 & 1 & \\
& 2 & 8434.2 & $\times$ & & & \\
\hline \hline & 8566.3 & 1 & & & \\
\hline
\end{tabular}

TABLE XLI. The partial width ratios for the $s c \bar{c} \bar{b}$ tetraquarks decay into $s \bar{b} \otimes c \bar{c}$ modes. For each state, we choose one mode as the reference channel, and the partial width ratios of the other channels are calculated relative to this channel. The masses are all in units of $\mathrm{MeV}$.

\begin{tabular}{ccccccc}
\hline \hline System & $J^{P}$ & Mass & $B_{s}^{*} J / \psi$ & $B_{s}^{*} \eta_{c}$ & $B_{s} J / \psi$ & $B_{s} \eta_{c}$ \\
\hline$s c \bar{c} \bar{b}$ & $0^{+}$ & 8220.1 & $\times$ & & & $\times$ \\
& & 8375.2 & $\times$ & & & 1 \\
& & 8488.1 & $\times$ & & & 1 \\
& & 8561.0 & 8.9 & & & 1 \\
& $1^{+}$ & 8287.8 & $\times$ & $\times$ & $\times$ & \\
& & 8368.4 & $\times$ & $\times$ & $\times$ & \\
& & 8407.5 & $\times$ & 1 & $\times$ & \\
& & 8498.8 & $\times$ & 1 & 0.1 & \\
& & 8531.2 & 0.02 & 1 & 5.2 & \\
& 8551.1 & 42.9 & 1 & 0.1 & \\
& 2 & 8434.2 & $\times$ & & & \\
\hline \hline
\end{tabular}

into the $q \bar{c} \otimes Q \bar{b}$ and $q \bar{b} \otimes Q \bar{c}$ configurations, as shown in Tables XXXII-XXXV. From these tables, we see that some eigenstates couple very strongly with two $S$-wave mesons. Taking $n b \bar{c} \bar{b}$ tetraquarks as example, the lowest eigenstate

$$
T\left(n b \bar{c} \bar{b}, 11293.4,0^{+}\right)=0.98341 \bar{D} \eta_{b}+\cdots .
$$

This state couples almost completely (96.7\%) to the $\bar{D} \eta_{b}$ scattering state. Thus it may be very broad and hid in the continuum. Note that this kind of state also exists in the calculation of the hidden charm tetraquarks and pentaquarks, where the lower mass state couples strongly to a heavy charmonium and a light hadron $[19,65,86]$. Moreover, the $T\left(n b \bar{c} \bar{b}, 11314.8,1^{+}\right)$ and $T\left(n b \bar{c} \bar{b}, 11392.4,1^{+}\right)$states couple very strongly to $\bar{D} \Upsilon$ and $\bar{D}^{*} \eta_{b}$ channels. Both the $T\left(n b \bar{c} \bar{b}, 11438.6,0^{+}\right)$, $T\left(n b \bar{c} \bar{b}, 11446.6,1^{+}\right)$and $T\left(n b \bar{c} \bar{b}, 11453.9,2^{+}\right)$couple very strongly to $\bar{D}^{*} \Upsilon$. They may be scattering states. To draw a definite conclusion, dynamical studies like the complex scaling methods $[87,88]$ are needed, which is beyond the present work. For clarity, we add a fifth column in Tables XXX-XXXI to indicate these scattering states.

We plot the relative position of the $q c \bar{c} \bar{b}$ and $q b \bar{c} \bar{b}$ tetraquarks in Figs. 5-6. For comparison, we also plot the possible scattering states. They are marked with a dagger $(\dagger)$, along with the proportion of their dominant components. We can easily see that they all lie close to the corresponding meson-meson thresholds.

After identifying the scattering states, the other states are genuine tetraquarks. They all lie far above their $S$-wave decay channel(s). In particular, the $q b \bar{c} \bar{b}$ tetraquarks lie above all possible thresholds of two $S$-wave mesons. Thus they may be broad states. We also study their decay properties, which can be found in Tables XXXVI-XLVII.

TABLE XLII. The values of $k \cdot\left|c_{i}\right|^{2}$ for the $n b \bar{c} \bar{b}$ tetraquarks (in units of MeV).

\begin{tabular}{|c|c|c|c|c|c|c|c|c|c|c|}
\hline \multirow[b]{2}{*}{ System } & \multirow[b]{2}{*}{$J^{P}$} & \multirow[b]{2}{*}{ Mass } & \multicolumn{4}{|c|}{$n \bar{c} \otimes b \bar{b}$} & \multicolumn{4}{|c|}{$n \bar{b} \otimes b \bar{c}$} \\
\hline & & & $\bar{D}^{*} \Upsilon$ & $\bar{D}^{*} \eta_{b}$ & $\bar{D} \Upsilon$ & $\bar{D} \eta_{b}$ & $B^{*} \bar{B}_{c}^{*}$ & $B^{*} \bar{B}_{c}$ & $B \bar{B}_{c}^{*}$ & $B \bar{B}_{c}$ \\
\hline \multirow[t]{12}{*}{$\overline{n b \bar{c} \bar{b}}$} & $0^{+}$ & 11253.4 & $x$ & & & $x$ & $x$ & & & $\times$ \\
\hline & & 11438.6 & $x$ & & & 6.5 & $x$ & & & $x$ \\
\hline & & 11673.1 & 109.1 & & & 11.5 & 9.5 & & & 432.4 \\
\hline & & 11748.7 & 0.3 & & & 18.2 & 543.5 & & & 42.7 \\
\hline & $1^{+}$ & 11314.8 & $x$ & $x$ & $x$ & & $\times$ & $x$ & $x$ & \\
\hline & & 11392.4 & $x$ & $x$ & 3.8 & & $x$ & $x$ & $x$ & \\
\hline & & 11446.6 & $x$ & 0.8 & 0.01 & & $x$ & $x$ & $x$ & \\
\hline & & 11697.5 & 75.4 & 3.1 & 2.1 & & 1.0 & 357.0 & 110.7 & \\
\hline & & 11719.1 & 2.7 & 38.6 & 18.0 & & 10.1 & 149.3 & 386.9 & \\
\hline & & 11744.9 & 0.8 & 7.4 & 13.7 & & 529.9 & 2.1 & 16.6 & \\
\hline & $2^{+}$ & 11453.9 & $x$ & & & & $\times$ & & & \\
\hline & & 11747.6 & 50.2 & & & & 499.3 & & & \\
\hline
\end{tabular}


TABLE XLIII. The values of $k \cdot\left|c_{i}\right|^{2}$ for the $s b \bar{c} \bar{b}$ tetraquarks (in units of MeV).

\begin{tabular}{|c|c|c|c|c|c|c|c|c|c|c|}
\hline \multirow[b]{2}{*}{ System } & \multirow[b]{2}{*}{$J^{P}$} & \multirow[b]{2}{*}{ Mass } & \multicolumn{4}{|c|}{$s \bar{c} \otimes b \bar{b}$} & \multicolumn{4}{|c|}{$s \bar{b} \otimes b \bar{c}$} \\
\hline & & & $\bar{D}_{s}^{*} \Upsilon$ & $\bar{D}_{s}^{*} \eta_{b}$ & $\bar{D}_{s} \Upsilon$ & $\bar{D}_{s} \eta_{b}$ & $B_{s}^{*} \bar{B}_{c}^{*}$ & $B_{s}^{*} \bar{B}_{c}$ & $B_{s} \bar{B}_{c}^{*}$ & $B_{s} \bar{B}_{c}$ \\
\hline \multirow[t]{12}{*}{$\overline{s b \bar{c} \bar{b}}$} & $0^{+}$ & 11355.0 & $x$ & & & $x$ & $x$ & & & $x$ \\
\hline & & 11539.4 & $x$ & & & 6.8 & $x$ & & & $x$ \\
\hline & & 11760.7 & 123.5 & & & 12.1 & 4.8 & & & 423.6 \\
\hline & & 11836.7 & 0.001 & & & 17.0 & 545.9 & & & 31.3 \\
\hline & $1^{+}$ & 11416.4 & $\times$ & $x$ & $x$ & & $x$ & $x$ & $x$ & \\
\hline & & 11496.1 & $x$ & $x$ & 3.4 & & $x$ & $x$ & $x$ & \\
\hline & & 11549.1 & $x$ & 1.3 & 0.01 & & $x$ & $x$ & $x$ & \\
\hline & & 11785.1 & 81.3 & 2.2 & 1.7 & & 4.9 & 330.0 & 118.7 & \\
\hline & & 11802.8 & 1.9 & 39.9 & 18.8 & & 8.7 & 165.6 & 360.9 & \\
\hline & & 11833.6 & 2.8 & 8.3 & 13.2 & & 519.6 & 0.1 & 21.7 & \\
\hline & $2^{+}$ & 11558.4 & $\times$ & & & & $\times$ & & & \\
\hline & & 11832.7 & 49.4 & & & & 484.7 & & & \\
\hline
\end{tabular}

TABLE XLIV. The partial width ratios for the $n b \bar{c} \bar{b}$ tetraquarks decay into $n \bar{c} \otimes b \bar{b}$ modes. For each state, we choose one mode as the reference channel, and the partial width ratios of the other channels are calculated relative to this channel. The masses are all in units of $\mathrm{MeV}$.

\begin{tabular}{ccccccc}
\hline \hline System & $J^{P}$ & Mass & $\bar{D}^{*} \Upsilon$ & $\bar{D}^{*} \eta_{b}$ & $\bar{D} \Upsilon$ & $\bar{D} \eta_{b}$ \\
\hline$n b \bar{c} \bar{b}$ & $0^{+}$ & 11253.4 & $\times$ & & & $\times$ \\
& & 11438.6 & $\times$ & & & 1 \\
& & 11673.1 & 9.5 & & & 1 \\
& & 11748.7 & 0.02 & & & 1 \\
& $1^{+}$ & 11314.8 & $\times$ & $\times$ & $\times$ & \\
& & 11392.4 & $\times$ & $\times$ & 1 & \\
& & 11446.6 & $\times$ & 59.0 & 1 & \\
& & 11697.5 & 35.6 & 1.5 & 1 & \\
& & 11719.1 & 0.1 & 2.1 & 1 & \\
& & 11744.9 & 0.1 & 0.5 & 1 & \\
& 2 & 11453.9 & $\times$ & & & \\
\hline \hline & & 11747.6 & 1 & & & \\
\hline
\end{tabular}

TABLE XLV. The partial width ratios for the $n b \bar{c} \bar{b}$ tetraquarks decay into $n \bar{b} \otimes b \bar{c}$ modes. For each state, we choose one mode as the reference channel, and the partial width ratios of the other channels are calculated relative to this channel. The masses are all in units of $\mathrm{MeV}$.

\begin{tabular}{ccccccc}
\hline \hline System & $J^{P}$ & Mass & $B^{*} \bar{B}_{c}^{*}$ & $B^{*} \bar{B}_{c}$ & $B \bar{B}_{c}^{*}$ & $B \bar{B}_{c}$ \\
\hline$n b \bar{c} \bar{b}$ & $0^{+}$ & 11253.4 & $\times$ & & & $\times$ \\
& & 11438.6 & $\times$ & & & $\times$ \\
& & 11673.1 & 0.02 & & & 1 \\
& & 11748.7 & 12.7 & & & 1 \\
& $1^{+}$ & 11314.8 & $\times$ & $\times$ & $\times$ & \\
& & 11392.4 & $\times$ & $\times$ & $\times$ & \\
& & 11446.6 & $\times$ & $\times$ & $\times$ & \\
& & 11697.5 & 0.01 & 3.2 & 1 & \\
& & 11719.1 & 0.03 & 0.4 & 1 & \\
& & 11744.9 & 31.9 & 0.1 & 1 & \\
& 2 & 11453.9 & $\times$ & & & \\
& & 11747.6 & 1 & & & \\
\hline \hline
\end{tabular}

TABLE XLVI. The partial width ratios for the $s b \bar{c} \bar{b}$ tetraquarks decay into $s \bar{c} \otimes b \bar{b}$ modes. For each state, we choose one mode as the reference channel, and the partial width ratios of the other channels are calculated relative to this channel. The masses are all in units of $\mathrm{MeV}$.

\begin{tabular}{ccccccc}
\hline \hline System & $J^{P}$ & Mass & $\bar{D}_{s}^{*} \Upsilon$ & $\bar{D}_{s}^{*} \eta_{b}$ & $\bar{D}_{s} \Upsilon$ & $\bar{D}_{s} \eta_{b}$ \\
\hline$s b \bar{c} \bar{b}$ & $0^{+}$ & 11355.0 & $\times$ & & & $\times$ \\
& & 11539.4 & $\times$ & & & 1 \\
& & 11760.7 & 10.2 & & & 1 \\
& & 11836.7 & 0.00003 & & & 1 \\
& $1^{+}$ & 11416.4 & $\times$ & $\times$ & $\times$ & \\
& & 11496.1 & $\times$ & $\times$ & 1 & \\
& & 11549.1 & $\times$ & 99.7 & 1 & \\
& 11785.1 & 47.8 & 1.3 & 1 & \\
& 11802.8 & 0.1 & 2.1 & 1 & \\
& & 11833.6 & 0.2 & 0.6 & 1 & \\
& 11558.4 & $\times$ & & & \\
& 2 & & & & & \\
\hline \hline
\end{tabular}

TABLE XLVII. The partial width ratios for the $s b \bar{c} \bar{b}$ tetraquarks decay into $s \bar{b} \otimes b \bar{c}$ modes. For each state, we choose one mode as the reference channel, and the partial width ratios of the other channels are calculated relative to this channel. The masses are all in units of $\mathrm{MeV}$.

\begin{tabular}{ccccccc}
\hline \hline System & $J^{P}$ & Mass & $B_{s}^{*} \bar{B}_{c}^{*}$ & $B_{s}^{*} \bar{B}_{c}$ & $B_{s} \bar{B}_{c}^{*}$ & $B_{s} \bar{B}_{c}$ \\
\hline$s b \bar{c} \bar{b}$ & $0^{+}$ & 11355.0 & $\times$ & & & $\times$ \\
& & 11539.4 & $\times$ & & & $\times$ \\
& & 11760.7 & 0.01 & & & 1 \\
& & 11836.7 & 17.4 & & & 1 \\
& $1^{+}$ & 11416.4 & $\times$ & $\times$ & $\times$ & \\
& & 11496.1 & $\times$ & $\times$ & $\times$ & \\
& & 11549.1 & $\times$ & $\times$ & $\times$ & \\
& & 11785.1 & 0.04 & 2.8 & 1 & \\
& & 11802.8 & 0.02 & 0.5 & 1 & \\
& & 11833.6 & 24.0 & 0.01 & 1 & \\
& $22^{+}$ & 11558.4 & $\times$ & & & \\
& & 11832.7 & 1 & & & \\
\hline \hline
\end{tabular}




\section{CONCLUSIONS}

Experimentally, lots of $X Y Z$ states have been found in this century. The singly charmed $X(2900)$ and doubly charmed $T_{c c}^{+}$were also observed recently. For all these system, the exchange of a light meson may be important since they are composed at least two light (anti)quarks. It is difficult to determine whether they are loosely bound molecular states or compact tetraquark states. The recently discovered $X(6900)$ 's are composed of four charm quarks. They are very likely compact tetraquarks since their interactions are mainly provided by gluon exchange. Similarly, if a triply heavy exotic state is observed in experiment, it is very likely a compact tetraquark.

In this work, we have systematically studied the triply heavy tetraquarks in an extended chromomagnetic model, which includes both colorelectric and chromomagnetic interactions. Our calculation suggests that the energy level is mainly determined by the color interaction. For the $q c \bar{c} \bar{c}$, $q b \bar{c} \bar{c}$ and $q b \bar{b} \bar{b}$ tetraquarks, the ground states are dominated by the $\sigma_{c} \otimes \bar{\sigma}_{c}$ component, which is similar to the fully heavy tetraquarks. However, the $q c \bar{b} \bar{b}$ tetraquarks are more like the doubly heavy tetraquarks, where the $6_{c} \otimes \bar{\sigma}_{c}$ component is mostly in the higher mass states, and the ground states are dominated by $\overline{3}_{c} \otimes 3_{c}$ components. We find no stable state which lie below the thresholds of two pseudoscalar mesons, in consistence with the results of Ref. [58]. The lowest axial-vector states with $q Q \bar{b} \bar{b}$ flavor configuration might be narrow because they lie just above the thresholds of two pseudoscalar mesons, but they cannot decay into these channels because of the conservation of the angular momentum and parity.

With the obtained wave functions, we also calculate the partial decay rates of the tetraquarks. We hope that our studies can be of help for the future experimental searches.

\section{ACKNOWLEDGMENTS}

This project was supported by the National Natural Science Foundation of China (NSFC) under Grants No. 11975033 and No. 12070131001.
[1] S. K. Choi et al. (Belle Collaboration), Observation of a Narrow Charmoniumlike State in Exclusive $B^{ \pm} \rightarrow K^{ \pm} \pi^{+} \pi^{-} J / \psi$ Decays, Phys. Rev. Lett. 91, 262001 (2003).

[2] D. Acosta et al. (CDF Collaboration), Observation of the Narrow State $X(3872) \rightarrow J / \psi \pi^{+} \pi^{-}$in $\bar{p} p$ Collisions at $\sqrt{s}=1.96 \mathrm{TeV}$, Phys. Rev. Lett. 93, 072001 (2004).

[3] V. M. Abazov et al. (D0 Collaboration), Observation and Properties of the $X(3872)$ Decaying to $J / \psi \pi^{+} \pi^{-}$in $p \bar{p}$ Collisions at $\sqrt{s}=1.96 \mathrm{TeV}$, Phys. Rev. Lett. 93, 162002 (2004).

[4] B. Aubert et al. (BABAR Collaboration), Study of the $B^{-} \rightarrow$ $J / \psi K^{-} \pi^{+} \pi^{-}$decay and measurement of the $B^{-} \rightarrow$ $X(3872) K^{-}$branching fraction, Phys. Rev. D 71, 071103 (2005).

[5] R. Aaij et al. (LHCb Collaboration), Observation of $X(3872)$ production in $p p$ collisions at $\sqrt{s}=7 \mathrm{TeV}$, Eur. Phys. J. C 72, 1972 (2012).

[6] S. Chatrchyan et al. (CMS Collaboration), Measurement of the $X(3872)$ production cross section via decays to $J / \psi \pi^{+} \pi^{-}$in $p p$ collisions at $\sqrt{s}=7 \mathrm{TeV}$, J. High Energy Phys. 04 (2013) 154.

[7] M. Ablikim et al. (BESIII Collaboration), Observation of $e^{+} e^{-} \rightarrow \gamma X(3872)$ at BESIII, Phys. Rev. Lett. 112, 092001 (2014).

[8] P. A. Zyla et al. (Particle Data Group), Review of particle physics, Prog. Theor. Exp. Phys. 2020, 083C01 (2020).

[9] M. Ablikim et al. (BESIII Collaboration), Observation of a Charged Charmoniumlike Structure in $e^{+} e^{-} \rightarrow \pi^{+} \pi^{-} J / \psi$ at $\sqrt{s}=4.26 \mathrm{GeV}$, Phys. Rev. Lett. 110, 252001 (2013).
[10] Z. Q. Liu et al. (Belle Collaboration), Study of $e^{+} e^{-} \rightarrow$ $\pi^{+} \pi^{-} J / \psi$ and Observation of a Charged Charmoniumlike State at Belle, Phys. Rev. Lett. 110, 252002 (2013); Erratum, Phys. Rev. Lett. 111, 019901 (2013).

[11] M. Ablikim et al. (BESIII Collaboration), Observation of a Charged $\left(D \bar{D}^{*}\right)^{ \pm}$Mass Peak in $e^{+} e^{-} \rightarrow \pi D \bar{D}^{*}$ at $\sqrt{s}=4.26 \mathrm{GeV}$, Phys. Rev. Lett. 112, 022001 (2014).

[12] M. Ablikim et al. (BESIII Collaboration), Confirmation of a charged charmoniumlike state $Z_{c}(3885)^{\mp}$ in $e^{+} e^{-} \rightarrow$ $\pi^{ \pm}\left(D \bar{D}^{*}\right)^{\mp}$ with double $D$ tag, Phys. Rev. D 92, 092006 (2015).

[13] M. Ablikim et al. (BESIII Collaboration), Observation of a Charged Charmoniumlike Structure $Z_{c}(4020)$ and Search for the $Z_{c}(3900)$ in $e^{+} e^{-} \rightarrow \pi^{+} \pi^{-} h_{c}$, Phys. Rev. Lett. 111, 242001 (2013).

[14] M. Ablikim et al. (BESIII Collaboration), Observation of a Charged Charmoniumlike Structure in $e^{+} e^{-} \rightarrow$ $\left(D^{*} \bar{D}^{*}\right)^{ \pm} \pi^{\mp}$ at $\sqrt{s}=4.26 \mathrm{GeV}$, Phys. Rev. Lett. 112, 132001 (2014).

[15] M. Ablikim et al. (BESIII Collaboration), Observation of a Near-Threshold Structure in the $K^{+}$Recoil-Mass Spectra in $e^{+} e^{-} \rightarrow K^{+}\left(D_{s}^{-} D^{* 0}+D_{s}^{*-} D^{0}\right)$, Phys. Rev. Lett. 126, 102001 (2021).

[16] R. Aaij et al. (LHCb Collaboration), Observation of New Resonances Decaying to $J / \psi K^{+}$and $J / \psi \phi$, Phys. Rev. Lett. 127, 082001 (2021).

[17] A. Bondar et al. (Belle Collaboration), Observation of Two Charged Bottomoniumlike Resonances in $\Upsilon(5 S)$ Decays, Phys. Rev. Lett. 108, 122001 (2012).

[18] L. Maiani, F. Piccinini, A. D. Polosa, and V. Riquer, Diquark-antidiquark states with hidden or open charm 
and the nature of $X(3872)$, Phys. Rev. D 71, 014028 (2005).

[19] Y. Cui, X.-L. Chen, W.-Z. Deng, and S.-L. Zhu, Possible Heavy Tetraquarks $q Q \bar{q} \bar{Q}, q q \bar{Q} \bar{Q}$ and $q Q \bar{Q} \bar{Q}$, High Energy Phys. Nucl. Phys. 31, 7 (2007).

[20] D. Ebert, R. N. Faustov, V. O. Galkin, and W. Lucha, Masses of tetraquarks with two heavy quarks in the relativistic quark model, Phys. Rev. D 76, 114015 (2007).

[21] W. Park and S. H. Lee, Color spin wave functions of heavy tetraquark states, Nucl. Phys. A925, 161 (2014).

[22] M. N. Anwar, J. Ferretti, and E. Santopinto, Spectroscopy of the hidden-charm $[q c][\bar{q} \bar{c}]$ and $[s c][\bar{s} \bar{c}]$ tetraquarks in the relativized diquark model, Phys. Rev. D 98, 094015 (2018).

[23] N. A. Törnqvist, From the deuteron to deusons, an analysis of deuteronlike meson-meson bound states, Z. Phys. C 61, 525 (1994).

[24] N. A. Törnqvist, Isospin breaking of the narrow charmonium state of Belle at $3872 \mathrm{MeV}$ as a deuson, Phys. Lett. B 590, 209 (2004).

[25] E. S. Swanson, Short range structure in the $X(3872)$, Phys. Lett. B 588, 189 (2004).

[26] C. Hanhart, Y.S. Kalashnikova, A. E. Kudryavtsev, and A. V. Nefediev, Reconciling the $X(3872)$ with the nearthreshold enhancement in the $D^{0} \bar{D}^{* 0}$ final state, Phys. Rev. D 76, 034007 (2007).

[27] T. F. Caramés, A. Valcarce, and J. Vijande, Charged charmonium molecules, Phys. Rev. D 82, 054032 (2010).

[28] F. Aceti, R. Molina, and E. Oset, $X(3872) \rightarrow J / \psi \gamma \gamma$ decay in the $D \bar{D} *$ molecular picture, Phys. Rev. D 86, 113007 (2012).

[29] R. Chen, X. Liu, Y.-R. Liu, and S.-L. Zhu, Predictions of the hidden-charm molecular states with the four quark components, Eur. Phys. J. C 76, 319 (2016).

[30] S.-L. Zhu, The possible interpretations of $Y(4260)$, Phys. Lett. B 625, 212 (2005).

[31] A. Esposito, A. Pilloni, and A. D. Polosa, Hybridized tetraquarks, Phys. Lett. B 758, 292 (2016).

[32] H.-X. Chen, W. Chen, X. Liu, and S.-L. Zhu, The hiddencharm pentaquark and tetraquark states, Phys. Rep. 639, 1 (2016).

[33] A. Esposito, A. Pilloni, and A. D. Polosa, Multiquark resonances, Phys. Rep. 668, 1 (2017).

[34] R. F. Lebed, R. E. Mitchell, and E. S. Swanson, Heavyquark QCD exotica, Prog. Part. Nucl. Phys. 93, 143 (2017).

[35] A. Ali, J. S. Lange, and S. Stone, Exotics: Heavy pentaquarks and tetraquarks, Prog. Part. Nucl. Phys. 97, 123 (2017).

[36] M. Karliner, J. L. Rosner, and T. Skwarnicki, Multiquark states, Annu. Rev. Nucl. Part. Sci. 68, 17 (2018).

[37] S. L. Olsen, T. Skwarnicki, and D. Zieminska, Nonstandard heavy mesons and baryons: Experimental evidence, Rev. Mod. Phys. 90, 015003 (2018).

[38] F.-K. Guo, C. Hanhart, U.-G. Meißner, Q. Wang, Q. Zhao, and B.-S. Zou, Hadronic molecules, Rev. Mod. Phys. 90, 015004 (2018).

[39] C.-Z. Yuan, The XYZ states revisited, Int. J. Mod. Phys. A 33, 1830018 (2018).
[40] Y.-R. Liu, H.-X. Chen, W. Chen, X. Liu, and S.-L. Zhu, Pentaquark and tetraquark states, Prog. Part. Nucl. Phys. 107, 237 (2019).

[41] N. Brambilla, S. Eidelman, C. Hanhart, A. Nefediev, C.-P. Shen, C. E. Thomas, A. Vairo, and C.-Z. Yuan, The $X Y Z$ states: Experimental and theoretical status and perspectives, Phys. Rep. 873, 1 (2020).

[42] V. M. Abazov et al. (D0 Collaboration), Evidence for a $B_{s}^{0} \pi^{ \pm}$State, Phys. Rev. Lett. 117, 022003 (2016).

[43] Y.-R. Liu, X. Liu, and S.-L. Zhu, X(5568) and its partner states, Phys. Rev. D 93, 074023 (2016).

[44] R. Aaij et al. (LHCb Collaboration), Search for Structure in the $B_{s}^{0} \pi^{ \pm}$Invariant Mass Spectrum, Phys. Rev. Lett. 117, 152003 (2016); 118, 109904(A) (2017).

[45] R. Aaij et al. (LHCb Collaboration), Model-Independent Study of Structure in $B^{+} \rightarrow D^{+} D^{-} K^{+}$Decays, Phys. Rev. Lett. 125, 242001 (2020).

[46] R. Aaij et al. (LHCb Collaboration), Amplitude analysis of the $B^{+} \rightarrow D^{+} D^{-} K^{+}$decay, Phys. Rev. D 102, 112003 (2020).

[47] R. Aaij et al. (LHCb Collaboration), Observation of an exotic narrow doubly charmed tetraquark, arXiv:2109 .01038 .

[48] R. Aaij et al. (LHCb Collaboration), Study of the doubly charmed tetraquark $T_{c c}^{+}$, arXiv:2109.01056.

[49] R. Aaij et al. (LHCb Collaboration), Observation of structure in the $J / \psi$-pair mass spectrum, Sci. Bull. 65, 1983 (2020).

[50] B. Silvestre-Brac, Systematics of $Q^{2}\left(\bar{Q}^{2}\right)$ systems with a chromomagnetic interaction, Phys. Rev. D 46, 2179 (1992).

[51] B. Silvestre-Brac and C. Semay, Systematics of $L=0 q^{2} \bar{q}^{2}$ systems, Z. Phys. C 57, 273 (1993).

[52] K. Chen, X. Liu, J. Wu, Y.-R. Liu, and S.-L. Zhu, Triply heavy tetraquark states with the $Q Q \bar{Q} \bar{q}$ configuration, Eur. Phys. J. A 53, 5 (2017).

[53] J.-F. Jiang, W. Chen, and S.-L. Zhu, Triply heavy $Q Q \bar{Q} \bar{q}$ tetraquark states, Phys. Rev. D 96, 094022 (2017).

[54] P. Junnarkar, N. Mathur, and M. Padmanath, Study of doubly heavy tetraquarks in Lattice QCD, Phys. Rev. D 99, 034507 (2019).

[55] Y. Liu, M. A. Nowak, and I. Zahed, Heavy holographic exotics: Tetraquarks as Efimov states, Phys. Rev. D 100, 126023 (2019).

[56] Y. Xing, Weak decays of triply heavy tetraquarks $b \bar{c} b \bar{q}$, Eur. Phys. J. C 80, 57 (2020).

[57] R. J. Hudspith, B. Colquhoun, A. Francis, R. Lewis, and K. Maltman, Lattice investigation of exotic tetraquark channels, Phys. Rev. D 102, 114506 (2020).

[58] Q.-F. Lü, D.-Y. Chen, Y.-B. Dong, and E. Santopinto, Triply-heavy tetraquarks in an extended relativized quark model, Phys. Rev. D 104, 054026 (2021).

[59] X.-Z. Weng, X.-L. Chen, and W.-Z. Deng, Masses of doubly heavy-quark baryons in an extended chromomagnetic model, Phys. Rev. D 97, 054008 (2018).

[60] H.-M. Chan, M. Fukugita, T. H. Hansson, H. J. Hoffman, K. Konishi, H. Hogaasen, and S. T. Tsou, Colour chemistryA study of metastable multiquark molecules, Phys. Lett. 76B, 634 (1978). 
[61] M. Fukugita, K. Konishi, and T. H. Hansson, Pseudobaryons, Phys. Lett. 74B, 261 (1978).

[62] K.-T. Chao, The $c \bar{c} q \bar{q}$ states, Nucl. Phys. B169, 281 (1980).

[63] K.-T. Chao, The $(c c)-(\bar{c} \bar{c})$ (Diquark-Antiqiquark) states in $e^{+} e^{-}$annihilation, Z. Phys. C 7, 317 (1981).

[64] H. Høgaasen, E. Kou, J.-M. Richard, and P. Sorba, Isovector and hidden-beauty partners of the $X(3872)$, Phys. Lett. B 732, 97 (2014).

[65] X.-Z. Weng, X.-L. Chen, W.-Z. Deng, and S.-L. Zhu, Hidden-charm pentaquarks and $P_{c}$ states, Phys. Rev. D 100, 016014 (2019).

[66] X.-Z. Weng, X.-L. Chen, W.-Z. Deng, and S.-L. Zhu, Systematics of fully heavy tetraquarks, Phys. Rev. D 103, 034001 (2021).

[67] X.-Z. Weng, W.-Z. Deng, and S.-L. Zhu, Doubly heavy tetraquarks in an extended chromomagnetic model, Chin. Phys. C 46, 013102 (2022).

[68] R. L. Jaffe, Multiquark hadrons. I. The phenomenology of $Q^{2} \bar{Q}^{2}$ mesons, Phys. Rev. D 15, 267 (1977).

[69] R. L. Jaffe, Multiquark hadrons. II. Methods, Phys. Rev. D 15, 281 (1977).

[70] A. De Rújula, H. Georgi, and S. L. Glashow, Hadron masses in a gauge theory, Phys. Rev. D 12, 147 (1975).

[71] F. Buccella, H. Høgaasen, J.-M. Richard, and P. Sorba, Chromomagnetism, flavour symmetry breaking and $S$-wave tetraquarks, Eur. Phys. J. C 49, 743 (2007).

[72] R. Aaij et al. (LHCb Collaboration), Observation of the Doubly Charmed Baryon $\Xi_{c c}^{++}$, Phys. Rev. Lett. 119, 112001 (2017).

[73] R. Aaij et al. (LHCb Collaboration), Precision measurement of the $\Xi_{c c}^{++}$mass, J. High Energy Phys. 02 (2020) 049.

[74] G.-J. Wang, L. Meng, and S.-L. Zhu, Spectrum of the fullyheavy tetraquark state $Q Q \bar{Q}^{\prime} \bar{Q}^{\prime}$, Phys. Rev. D 100, 096013 (2019).

[75] C. Deng, H. Chen, and J. Ping, Towards the understanding of fully-heavy tetraquark states from various models, Phys. Rev. D 103, 014001 (2021).
[76] R. Aaij et al. (LHCb Collaboration), Observation of $J / \psi p$ Resonances Consistent with Pentaquark States in $\Lambda_{b}^{0} \rightarrow$ $J / \psi K^{-} p$ Decays, Phys. Rev. Lett. 115, 072001 (2015).

[77] R. Aaij et al. (LHCb Collaboration), Observation of a Narrow Pentaquark State, $P_{c}(4312)^{+}$, and of the Two-Peak Structure of the $P_{c}(4450)^{+}$, Phys. Rev. Lett. 122, 222001 (2019).

[78] Q.-F. Lü, D.-Y. Chen, and Y.-B. Dong, Masses of fully heavy tetraquarks $Q Q \bar{Q} \bar{Q}$ in an extended relativized quark model, Eur. Phys. J. C 80, 871 (2020).

[79] D. Strottman, Multiquark baryons and the MIT bag model, Phys. Rev. D 20, 748 (1979).

[80] C. Gao, Group Theory and its Application in Particle Physics (in Chinese) (Higher Education Press, Beijing, China, 1992).

[81] E. Eichten, K. Gottfried, T. Kinoshita, K. D. Lane, and T.-M. Yan, Charmonium: The model, Phys. Rev. D 17, 3090 (1978); Erratum, Phys. Rev. D 21, 313 (1980).

[82] E. Eichten, K. Gottfried, T. Kinoshita, K. D. Lane, and T. M. Yan, Charmonium: Comparison with experiment, Phys. Rev. D 21, 203 (1980).

[83] S. Godfrey and N. Isgur, Mesons in a relativized quark model with chromodynamics, Phys. Rev. D 32, 189 (1985).

[84] J.-M. Richard, A. Valcarce, and J. Vijande, Hall-Post inequalities: Review and application to molecules and tetraquarks, Ann. Phys. (Amsterdam) 412, 168009 (2020).

[85] J.-M. Richard, A. Valcarce, and J. Vijande, Effect of relativistic kinematics on the stability of multiquarks, Phys. Rev. D 103, 054020 (2021).

[86] H. Høgaasen, J. M. Richard, and P. Sorba, Chromomagnetic mechanism for the $\mathrm{X}(3872)$ resonance, Phys. Rev. D 73, 054013 (2006).

[87] T. Myo and K. Kato, Complex scaling: Physics of unbound light nuclei and perspective, Prog. Theor. Exp. Phys. 2020, 12A101 (2020).

[88] G. Yang, J. Ping, and J. Segovia, Tetra- and penta-quark structures in the constituent quark model, Symmetry 12, 1869 (2020). 